\title{
تحقيق الاصالة في مشروعات إعادة الإعمار لمواقع التراث العالمي الثقافي المتضررة من الحروب والنزاعات
}

\author{
م/ فيفي عبد الله محمد مرسي \\ بكالوريوس الهندسة المعمارية \\ جامعة المنيا
}

Farida_2050y@yahoo.com

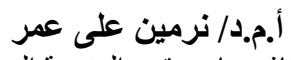 \\ أستاذ مساعد بقسم الهندسة المعمارية عمدية \\ جامعة طنطا
}

nermeen_omar@f-eng.tanta.edu.eg

\author{
أ.م.د /ايمن جمال الدين عبد التواب

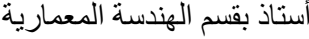

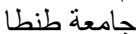

ayman.abdeltawab@f-eng.tanta.edu.eg
أولاً: الدراسة النظرية الوصفية والتي تناولت دراسة بعض المفاهيم الخاصة

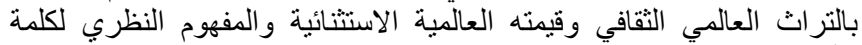

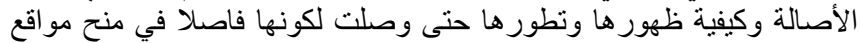

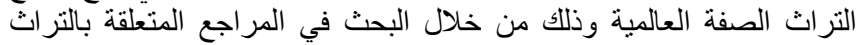

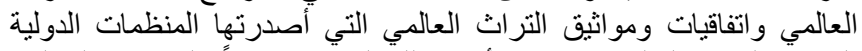

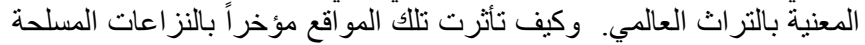

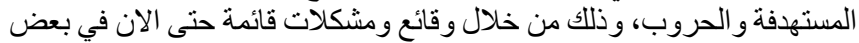

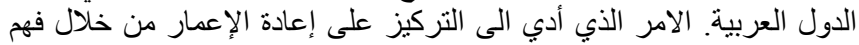

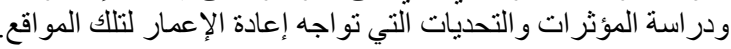

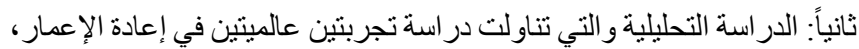

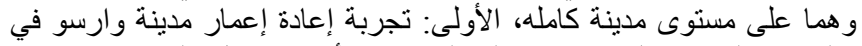

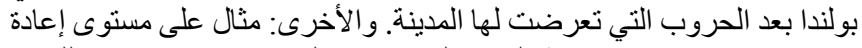

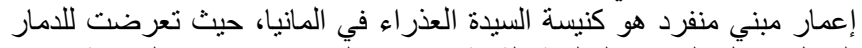

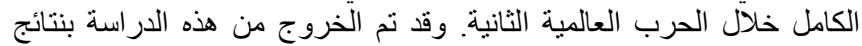

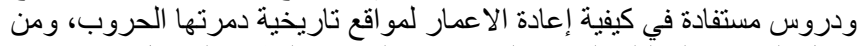

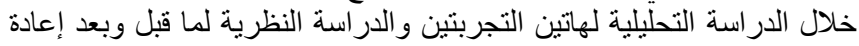

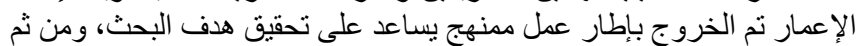
الخروج بالنتائج وصياغة التوصيات.

\section{الأدبيات المرجعية}

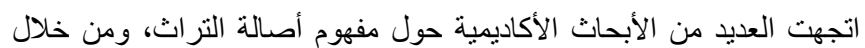

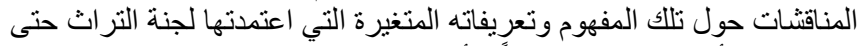

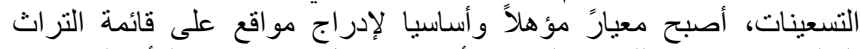

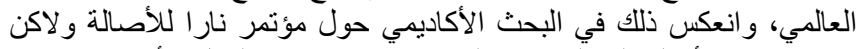

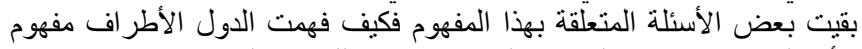

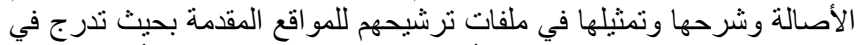

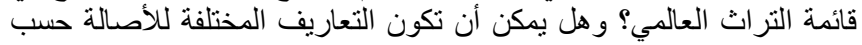

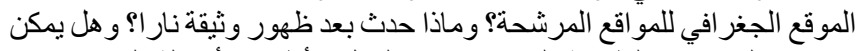

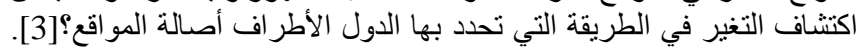

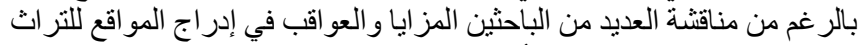

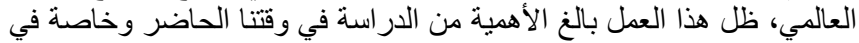

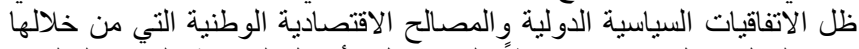

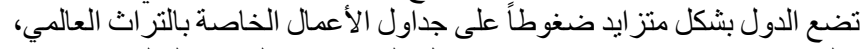

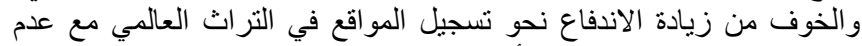

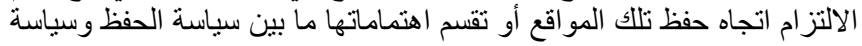

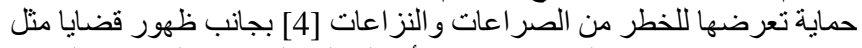

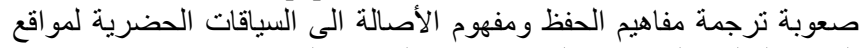

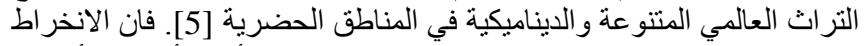

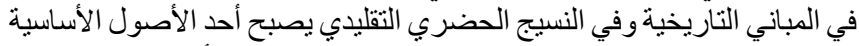

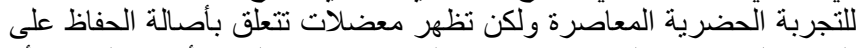

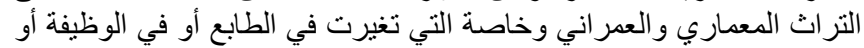

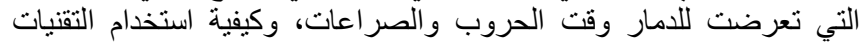

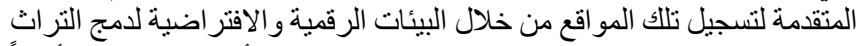

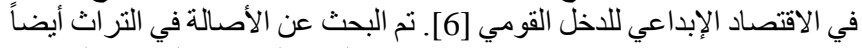

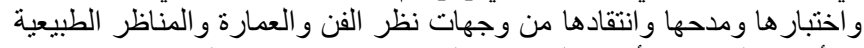

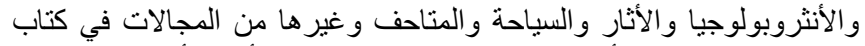

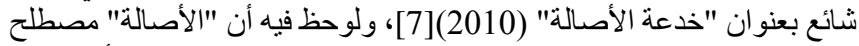

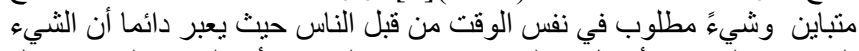

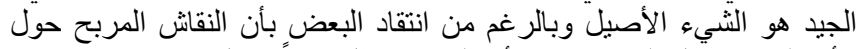

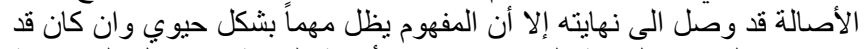

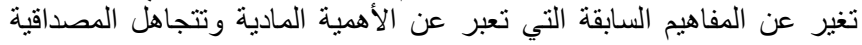

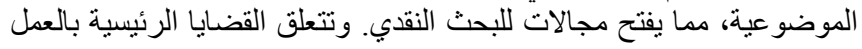

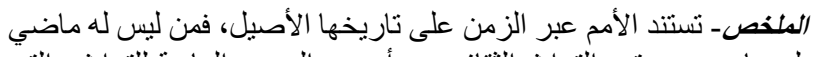

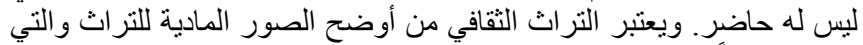

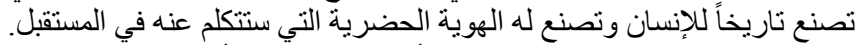

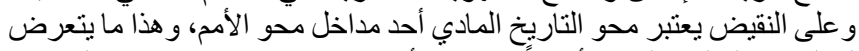

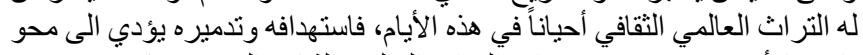

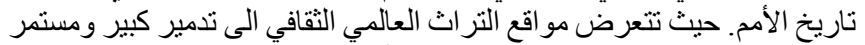

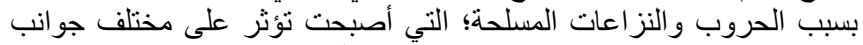

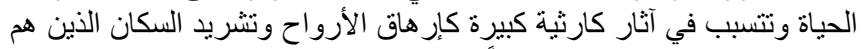

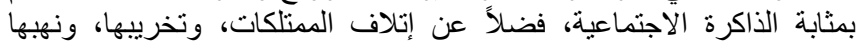

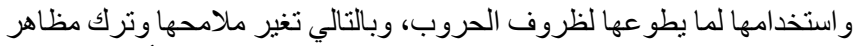

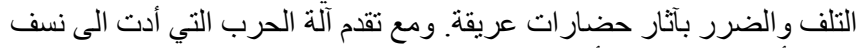

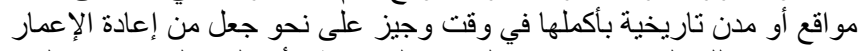

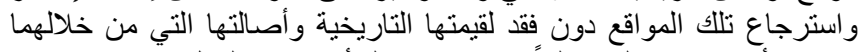

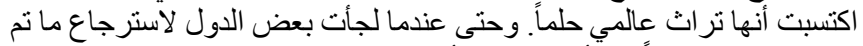

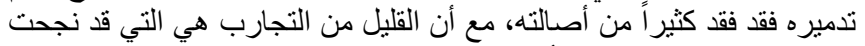

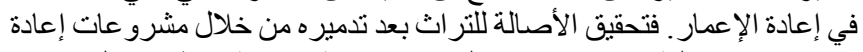

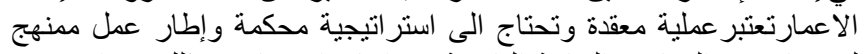

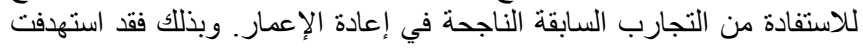

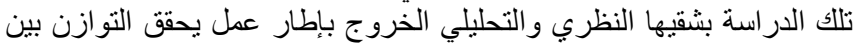

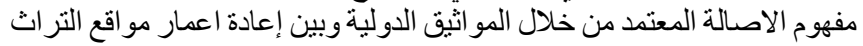

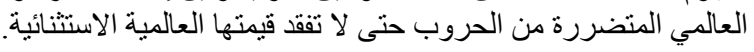

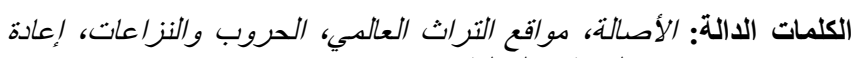
الإعمار، الاتفاقيات والمواثيق الألماتهولية.

المقدمة

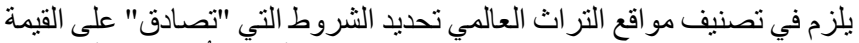

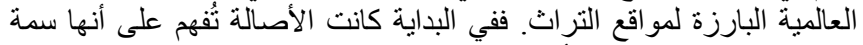

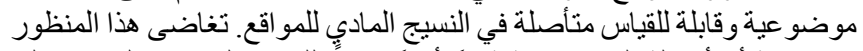

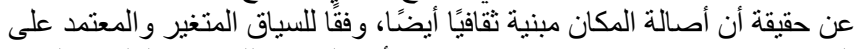

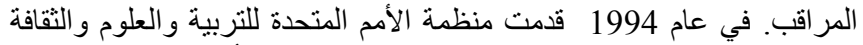

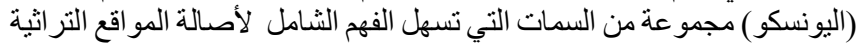

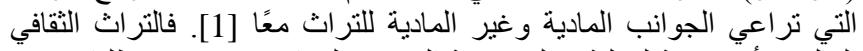

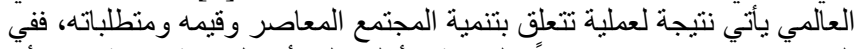

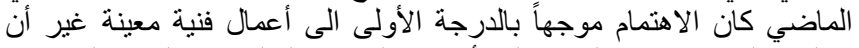

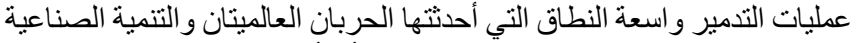

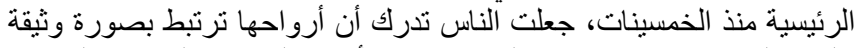

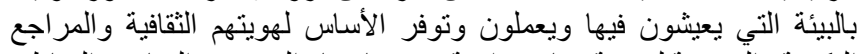

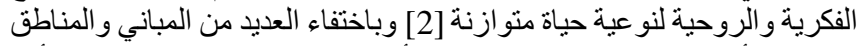

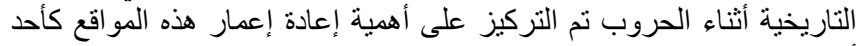

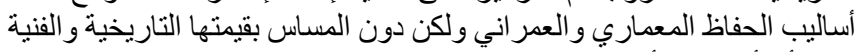

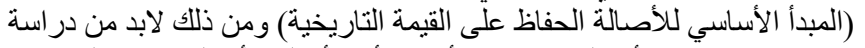

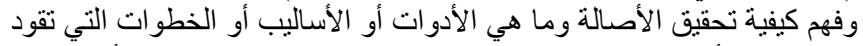

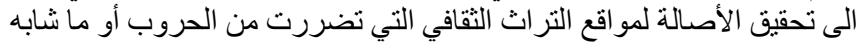

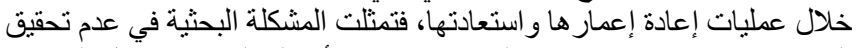

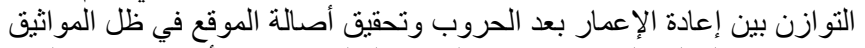

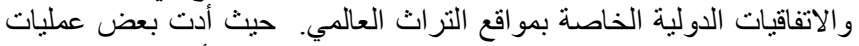

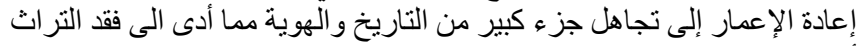

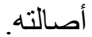

\section{منهجية البحث}

اعتمد البحث على نو عين من الدراسة هما: 
الأوروبية مثل الفرنسية، الإنجليزية والألمانية ولهذا فان اغلب الأوروبيين

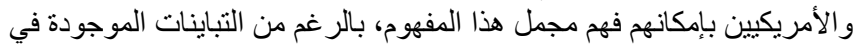

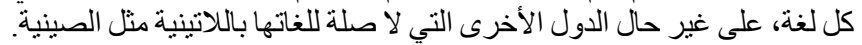

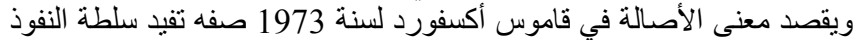

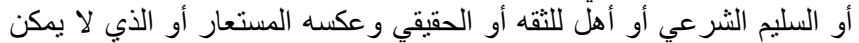

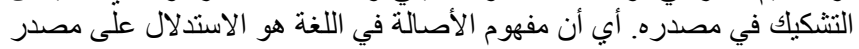

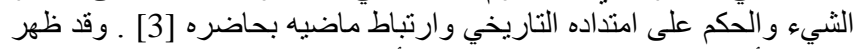

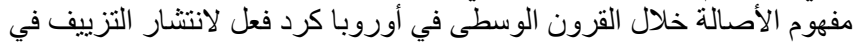

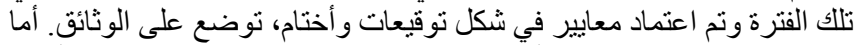

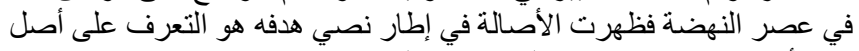

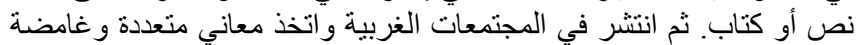

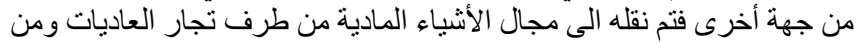

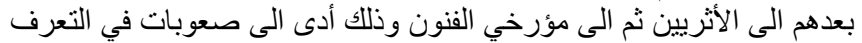

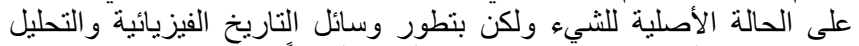

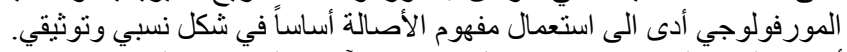

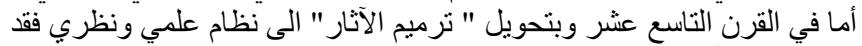

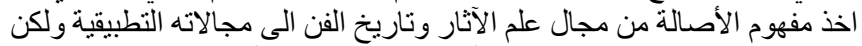

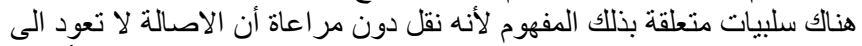

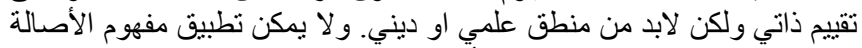

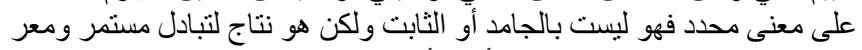

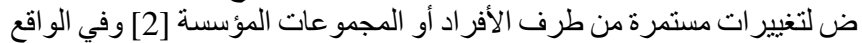

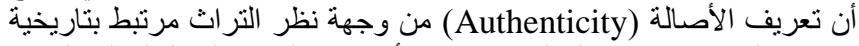

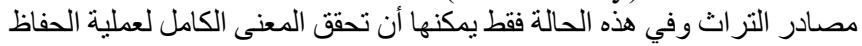

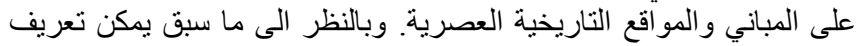

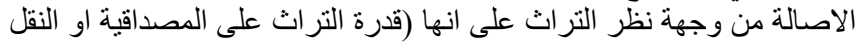

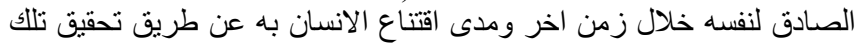

(المصداقية).

\section{2-2-1 المواثيق الدولية التي تتناول مفهوم الأصالة}

ميثاق فينيسيا (1964)

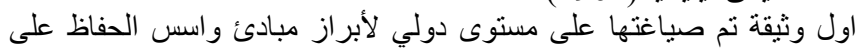

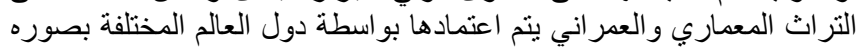

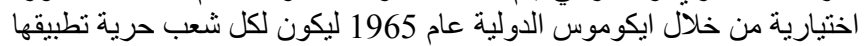

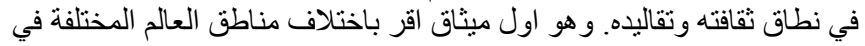

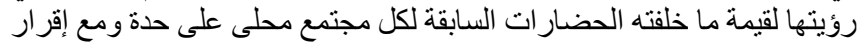

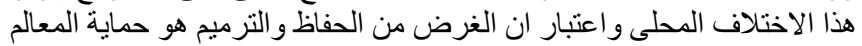

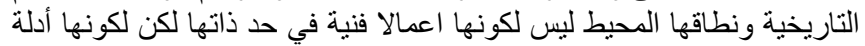

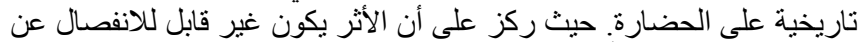

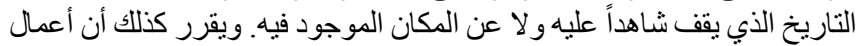

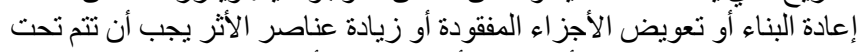

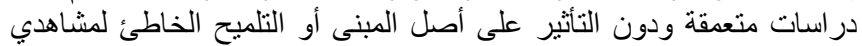

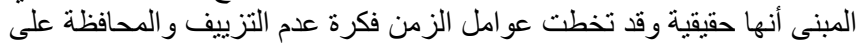

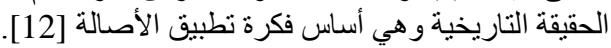

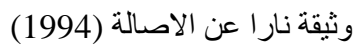

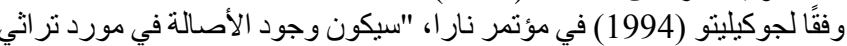

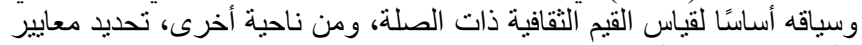

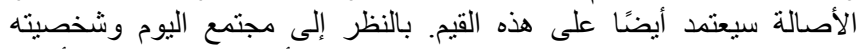

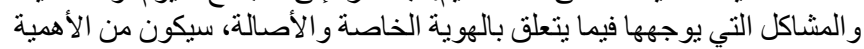

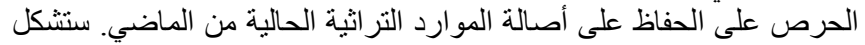

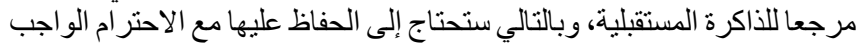

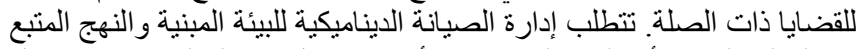

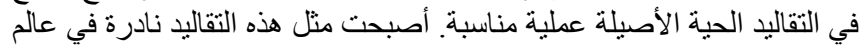

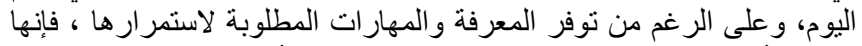

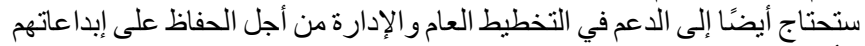

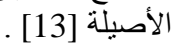

بنيت هذه الوثيقة على الجدلية عن حقيقة التراث والارتباط بالأصل او الاصيالة

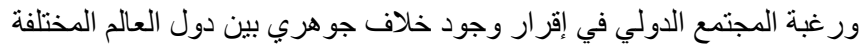

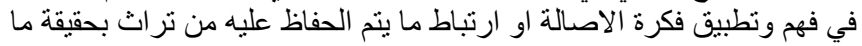

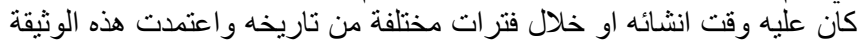

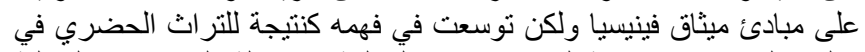

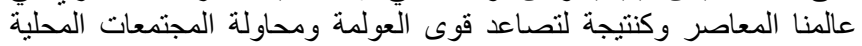

الذي يقوم به التراث في الظروف الخاصة والتي ينم سنه فيها وبالتالي يستلزم

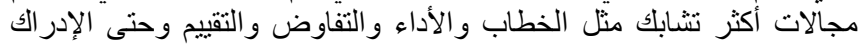

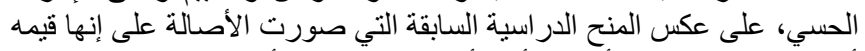

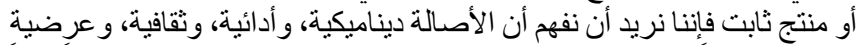

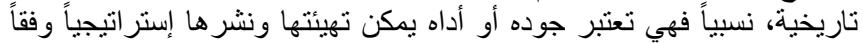

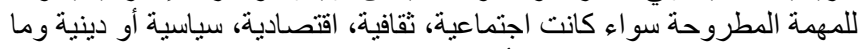

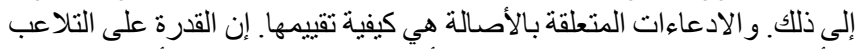

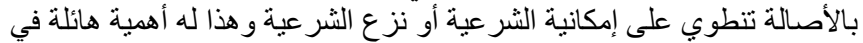

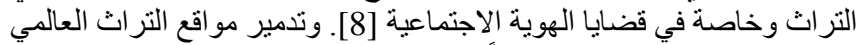

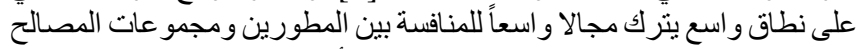

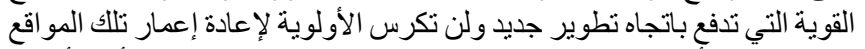

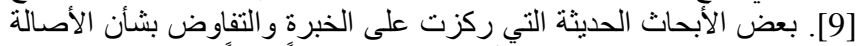

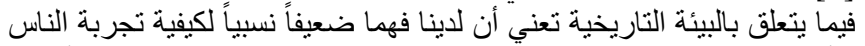

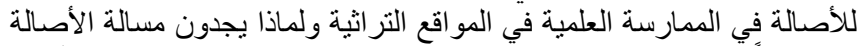

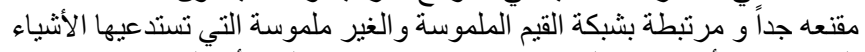

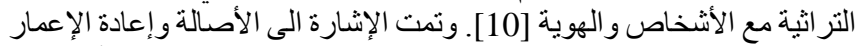

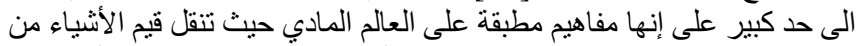

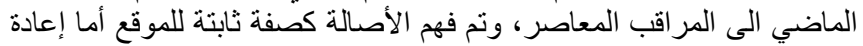

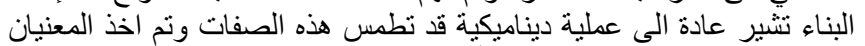

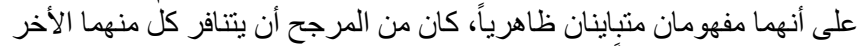

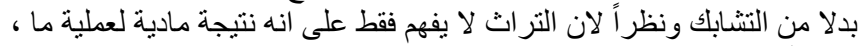

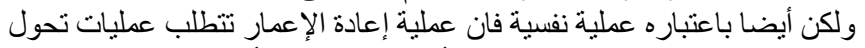

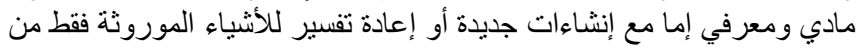

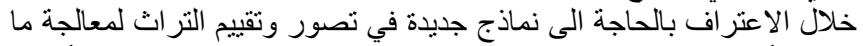

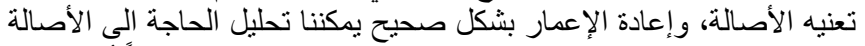

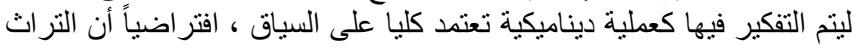

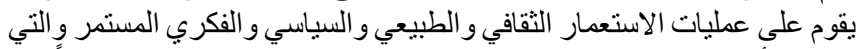

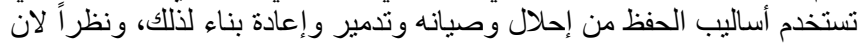

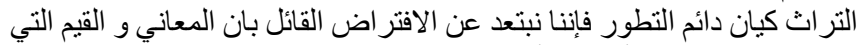

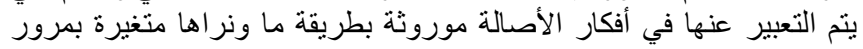

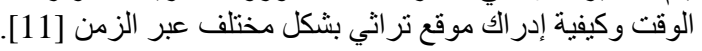

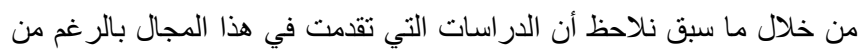

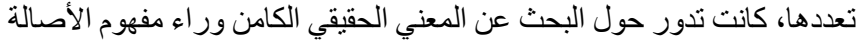

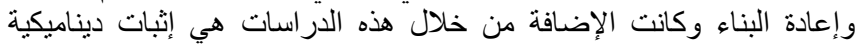

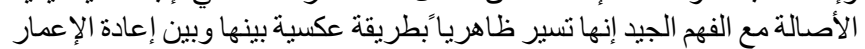

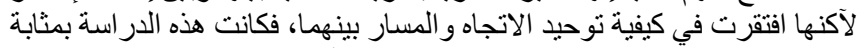

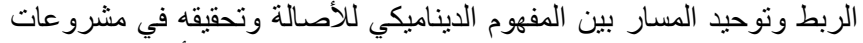
إعادة الإعمار لمو اقع التراث الثين العالمي بعد الحروب حتى لاني لا تفقد أصآلتها.

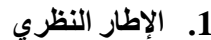

تناول الإطار النظري شرح بعض المفاهيم الهامة المكونة للبحث وعلافتها

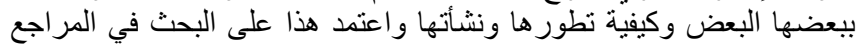

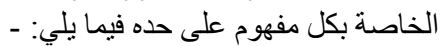

$$
\text { 1-1 التراث العالمي الثقافي }
$$

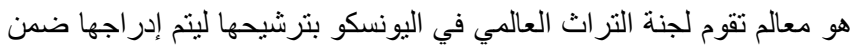

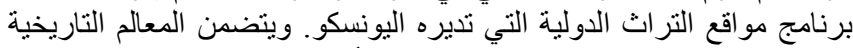

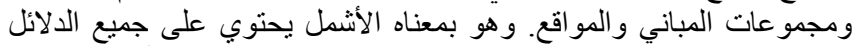

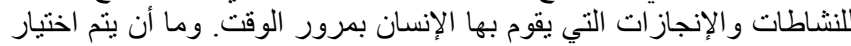

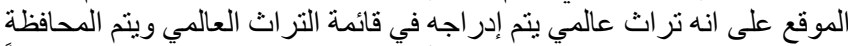

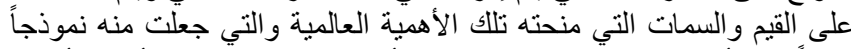

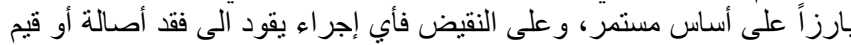

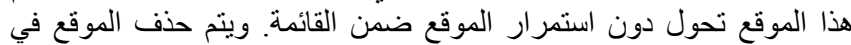

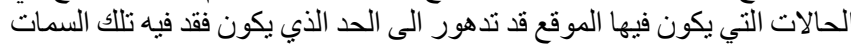

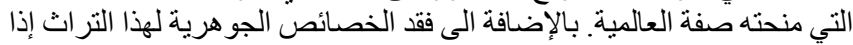

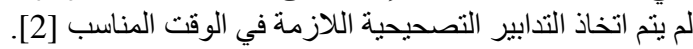

2-1 مفهوم الأصالة (Authenticity)

1-2-1 مفهوم الأصالة من واقع التاريخ واللغة

الأصالة كلمه صعبة الإحاطة ذات أصل يوناني لاتيني، عاثت في في اللغات 


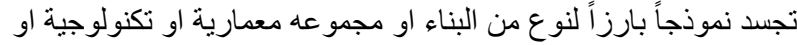
لمنظر يجسد فتره معبره لتاريخ الإنسانية.

تمثل نموذجا بارز التجمع بشري او شغل مجال إقليمي يجسد ثقافة او ثقافات خاصة إذا ما كان مهدد بتأثنير تغيير ات ات اكيدة.

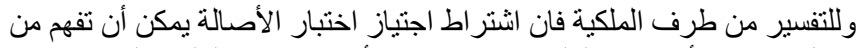

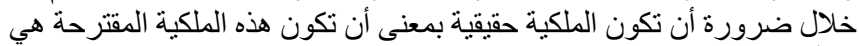

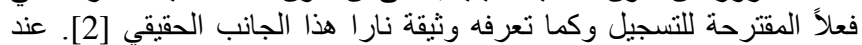

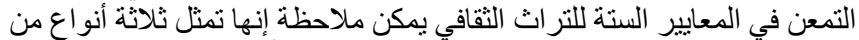

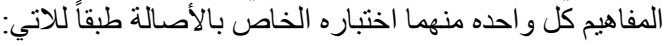

المعيار الأول: القبول في اختبار الاصلالة يعني ان الممتلكات المقترحة فيها دلالة الإنة

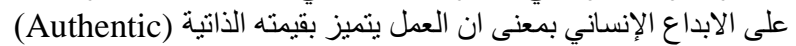

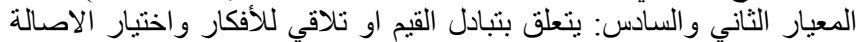

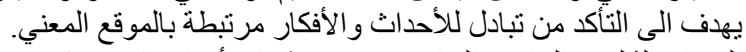

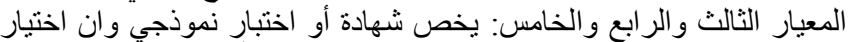

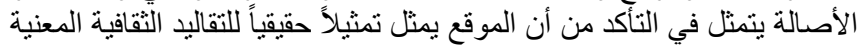
أو مثنال ملائم للنموذج البنائي أو شخل الأن الإقليم [2].

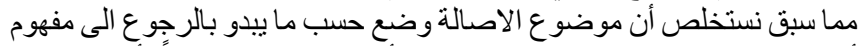

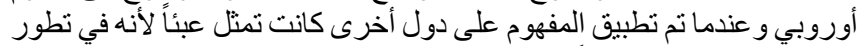

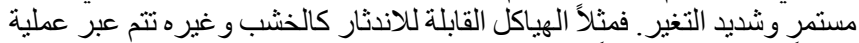

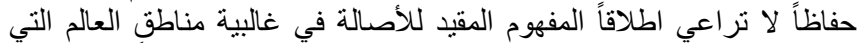

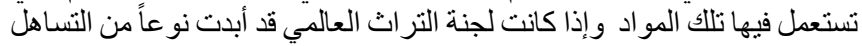

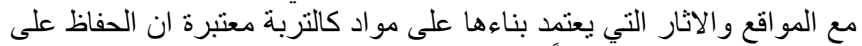

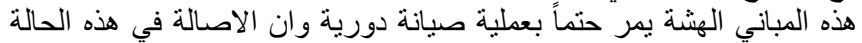

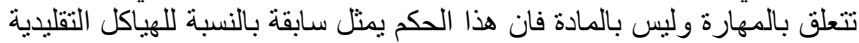

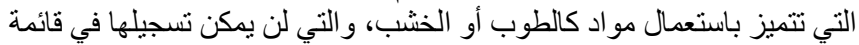
التراث العالمي بالنظر الى التقبد الحرفي بهذا المعيار الأوروبي للأصالة لئي

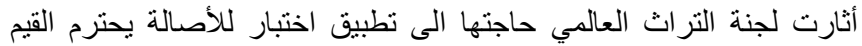

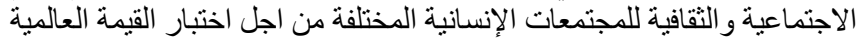

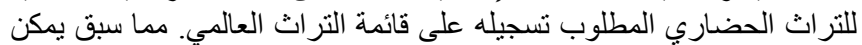

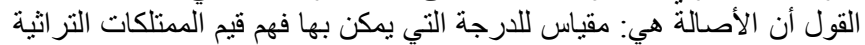

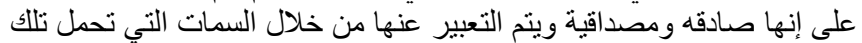

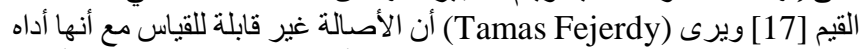

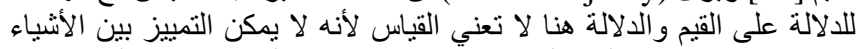

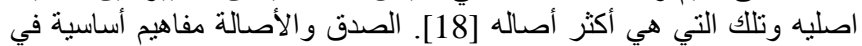

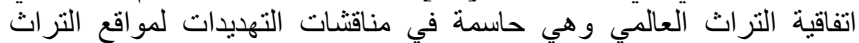

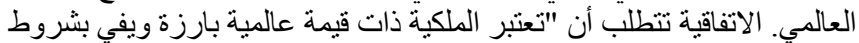

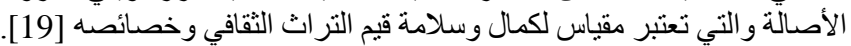

3-1 إعادة الإعمار

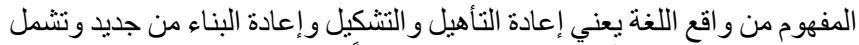

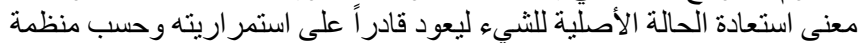

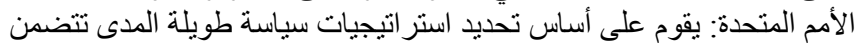

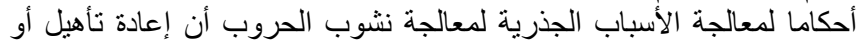

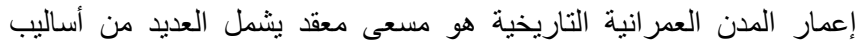

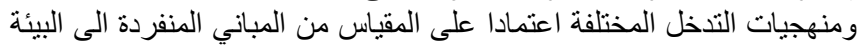

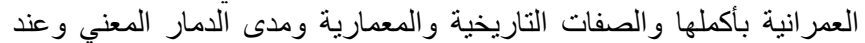

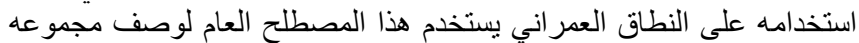

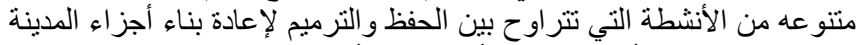

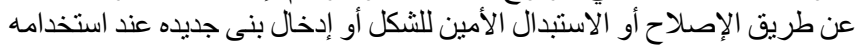

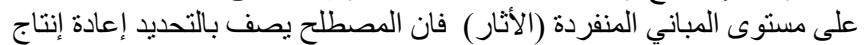

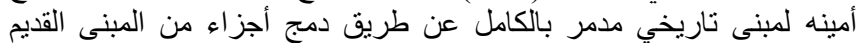

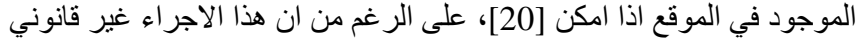

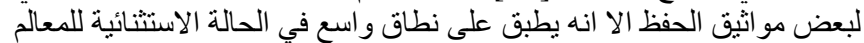

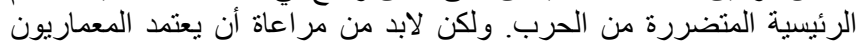

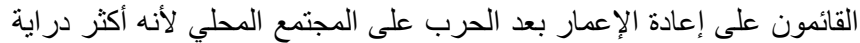

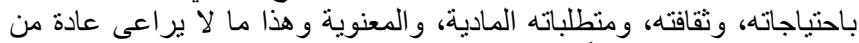

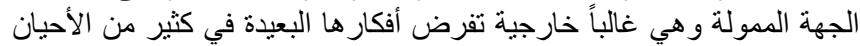

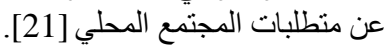

التأكيد على هويتها القومية. وأقرت الوثثية ان عمليات الحفاظ على التراث

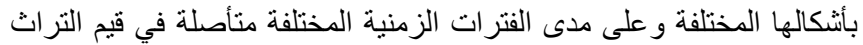

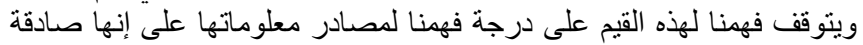
وموثوق بها.

وتكون الأصالة من خلال هذا الفهم بل يكون أساس في توجيه كل الدراسات

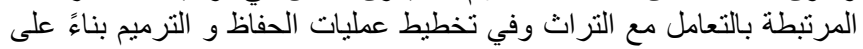

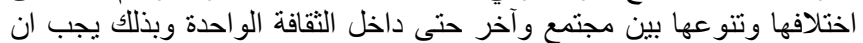

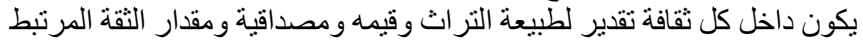

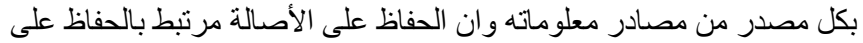

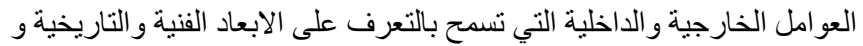

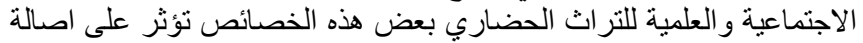

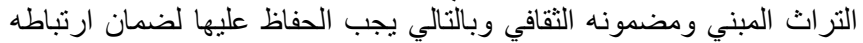

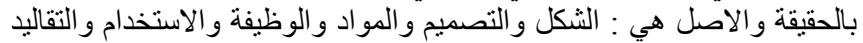

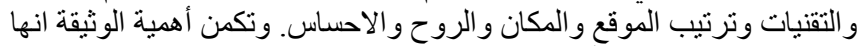

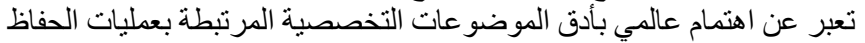

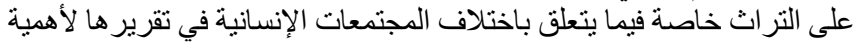

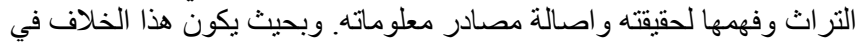
الفهم وتتوع التر اث .ومكوناته و أنشكاله سبيا في إثراء الحضاء الحضارة الإنسانية واحد

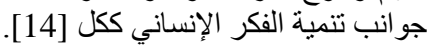

$$
\text { ميثاق و اشنطن } 1987
$$

مؤتمر الحفاظ على المدن التاريخية والمناطق العمر انية المنعقد في و اشنطن في

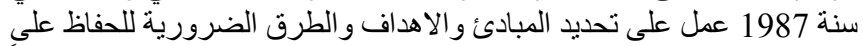

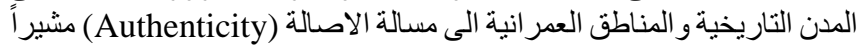

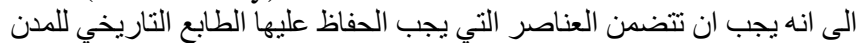

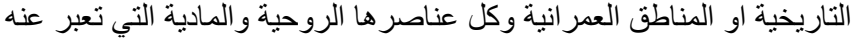

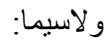

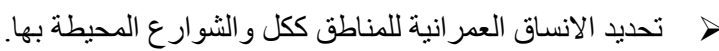

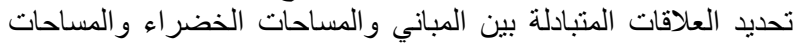

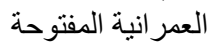

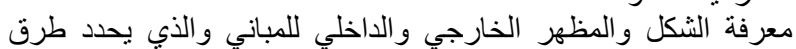

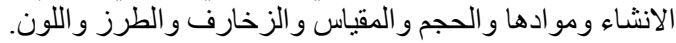

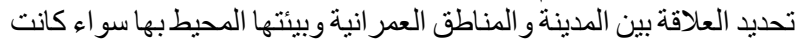
طبيعية او صناعية. المظاهر المختلفة التي اكتسبتها المناطق العمرانية مع مرور الزئة الزمان المساس بهذه القيم من شانه يؤثر على أصالة المدينة التاريخية أو المنطقة العنة

العمر انية [15].

الميثاق الدولي للسياحة الثقافية

أكد على أهمية الحفاظ على اصالة المجموعات التر اثية باعتبار ها شرطا لهاء أساسيا

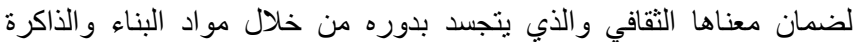

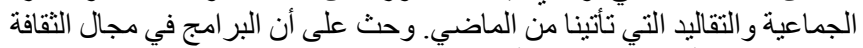

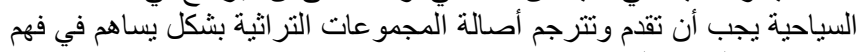

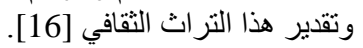

\section{3-2-1 الأصالة والتراث العالمي}

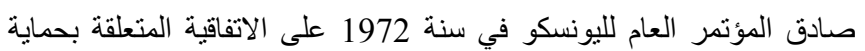

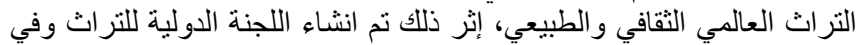

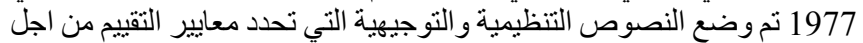

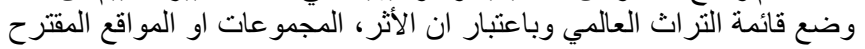

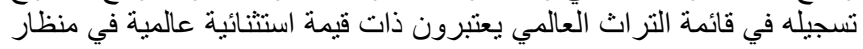

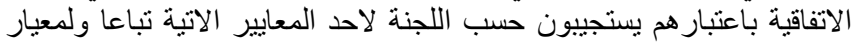
الاصالة. حيث ان أي موقع تر اث يجب اثب ان تستجيب الى انها: عمل إبداعي يجسد العبقرية الخلاقة للإنسان.

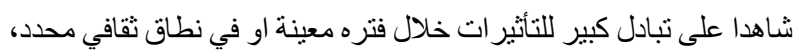

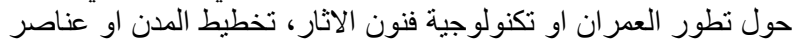
تنسيق المو اقع الطبيعية.

شاهدا وحيدا أو استثنائيا حول تقاليد ثقافية او حضارية حية او ميتة. 
3-3-1 معوقات إعادة الاعمار بعد الحروب

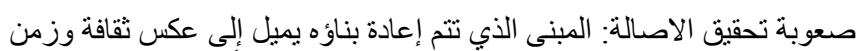

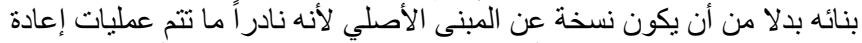

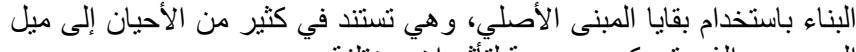
المصممين الذي قد يكون عرضئة لتأثير ات مختلفئة

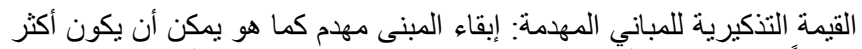

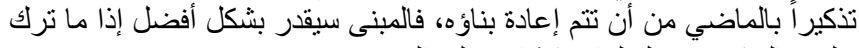
على حاله كمصدر للإلهام وكثاهد على الحدث.

النقل الخاطئ: نقل معلومات خاطئة تؤدي الى إعادة بناء غير دقيقة وتضليل

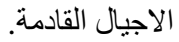

تدمير الادلة الاصلية: في العديد من عمليات إعادة البناء يتعذر الوصول إلى إلى الأدلة

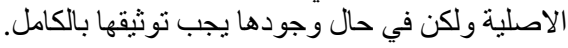

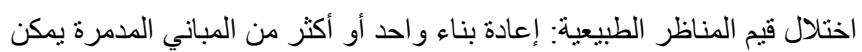

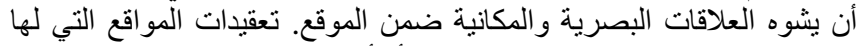

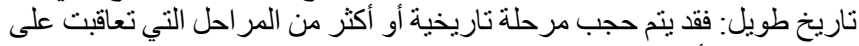

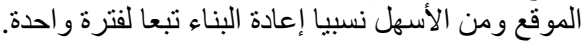

التكلفة: مشاريع إعادة الإعمار تميل إلى أن تكون مكلفة للغاية [25].

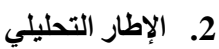

تجارب بعض الدول لتحقيق الاصلالة في مشروعات إعادة الاعمار. تم اختيار

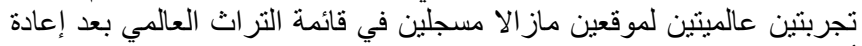

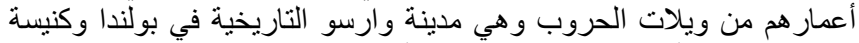

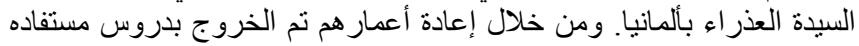
ساعدت على نجاح تحقيق إعادة الاعمار.

\section{1-2 تجربة مدينة وارسو البولندية بعد الحرب العالمية الثانية}

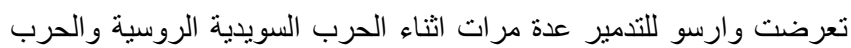

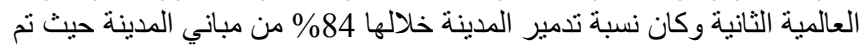
تدمير (782) مبنى بالكامل بجانب (141) مبنى بشكل جزئي وتمت العانية استر اتيجية إعادة الاعمار على النحو الاتي: -

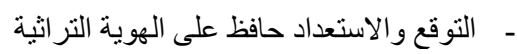

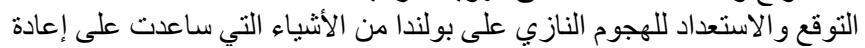

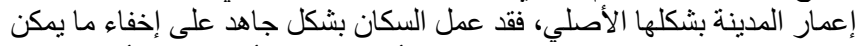

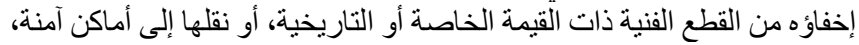

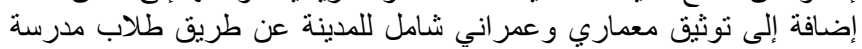

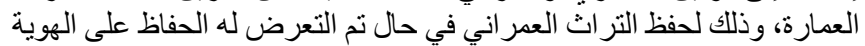

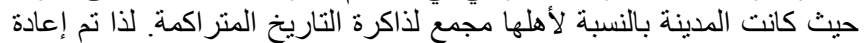

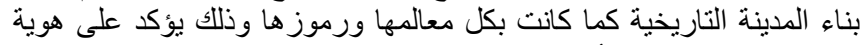

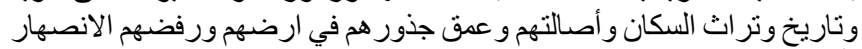
بأي هوية جديدة تفرض علئرن

- مفظ الذاكرة الجماعية

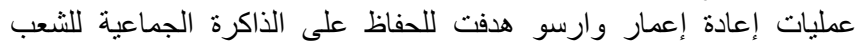

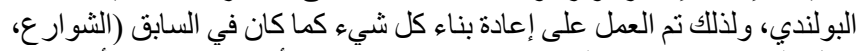

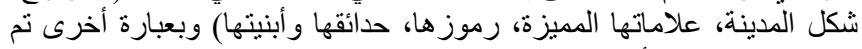

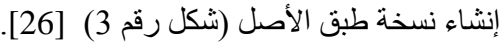

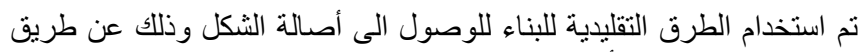

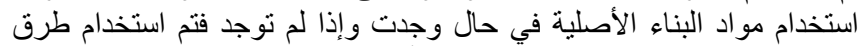

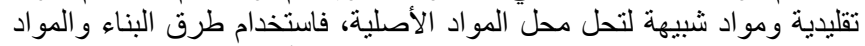

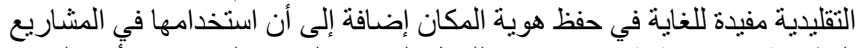

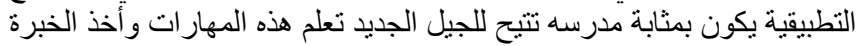

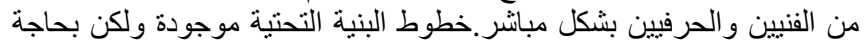

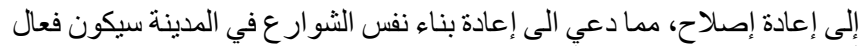

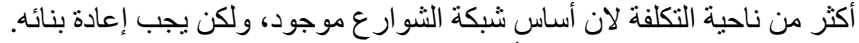
توفير سبل الر احة والحاجات الأساسية من خلانل إعادة بناء الواجهات التهات الخارجية

\section{1-3-1 مفهوم إعادة إعمار المباني التاريخية كما ورد في المواثيق الدولية}

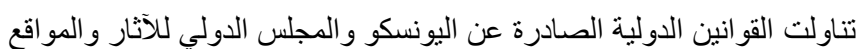

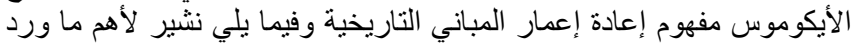

فيها :

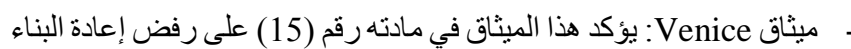
بالنسبة للأطلال الأثرية المكتشفة في الحفائر.

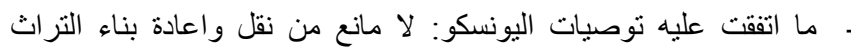

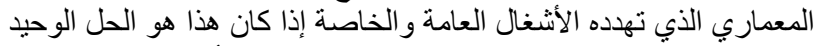

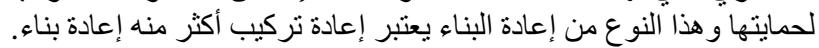

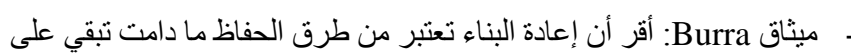

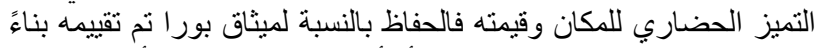

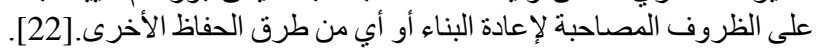

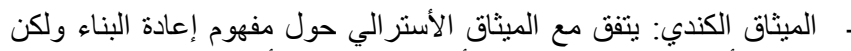
يضيف أنه عملية إعادة خلق كلي أو جزئي للمبنى الأثري بغرض الفي إلى إعادة إحياء القيم الثقافية للمبنى.

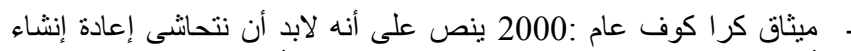

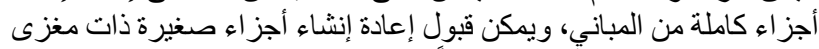

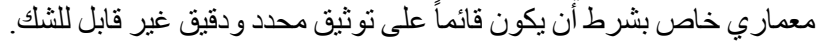
ميثاق واشنطن: ينص على احترام المخطط الفراغي للبناء فيما يتعلق بالمقياس و الحجم و المساحة [15] لمنئ - إعلان درسدن عام 1982 وتتلخص نتائجه في: • أن إعمار الحجر هو نشاط متكامل مع عملية إعمار البشر وليس نقيضاً لها أو بديلاً.

القيمة الوطنية لعملية إعادة الإعمار تعد تعميقاً للانتماء المشترك.

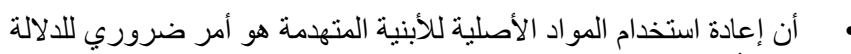
على أصالتها.

أن أعمال إعادة الإعمار تتضمن مرونة تمكن متخذي القرار من التصرف بموجبها.

• أن هناك حدوداً يجب ألا يتم تجاوزها في التعامل مع المناطق التاريخية

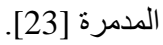

\section{2-3-1 مؤثرات عمليات إعادة الإعمار}

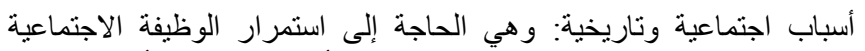

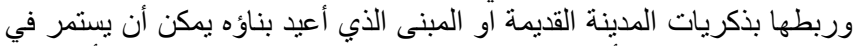

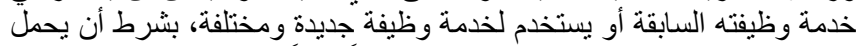

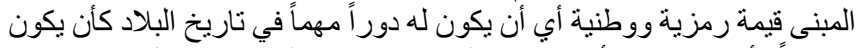

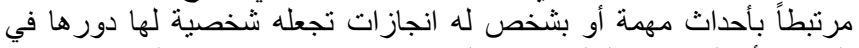

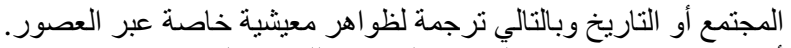

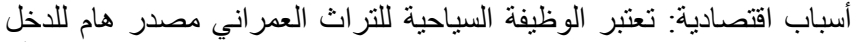

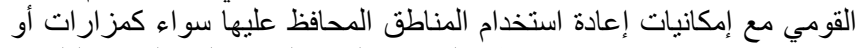

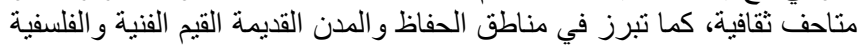

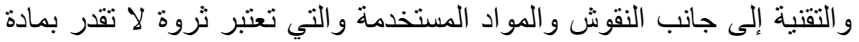
تقابلها. أسباب سياسية: حيث يتم من خلال الحفاظ التعلم من الماضي وسرد التاريخ

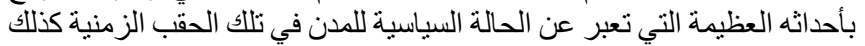

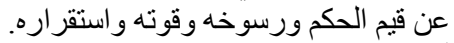

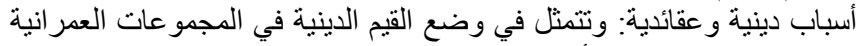

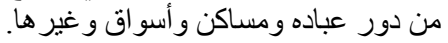

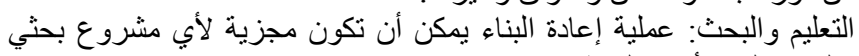
و المبنى الناتج أداة تعليمية مهمه.

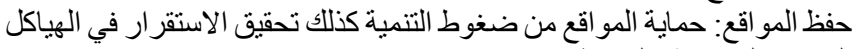
المدمرة المحفوفة بالمخاطر [24] 
الانتقائية: تم الحفاظ على جزء معين من التاريخ و إهمال حقابات أخرى، فكان

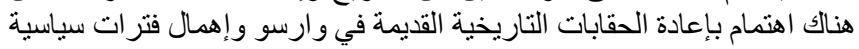

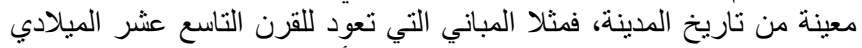

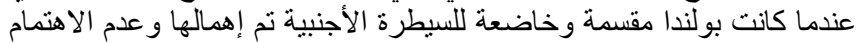

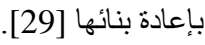

سلبيات وعو ائق عملية إعادة الإعمار في تجربة وارسو:

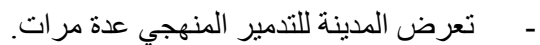

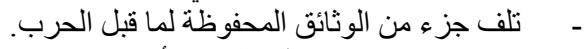
- - - - - -

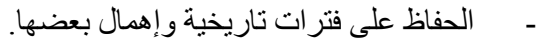

إيجابيات إعادة الإعمار : - إن

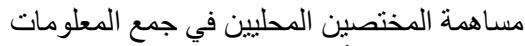

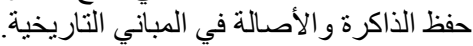

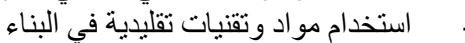

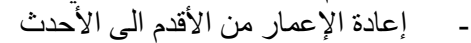

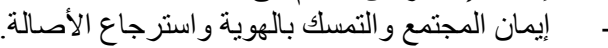

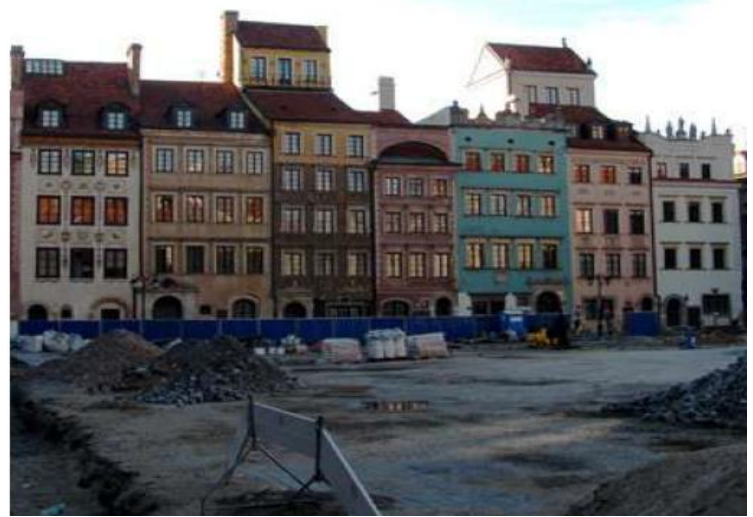

شكل 3 المباني في مدينة وارسو بعد إعادة الإعمار.

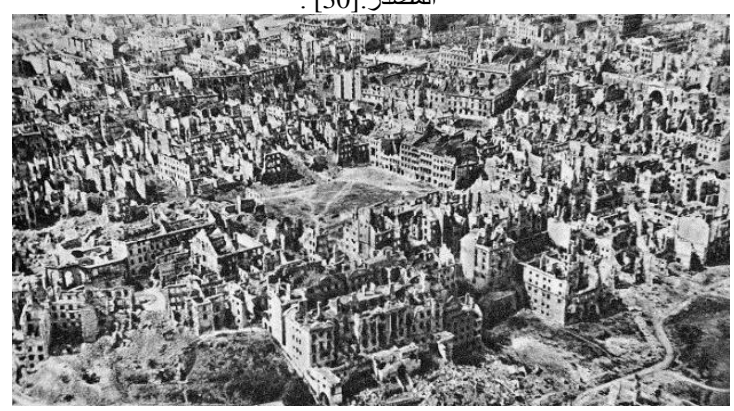

ثكل 4 الساحة الرئيسية لمدينة وارسو أثناء الحرب - المصدر:

https://commons.wikimedia.org/wiki/File:Warsaw_Old_Town_ 1945.jpg

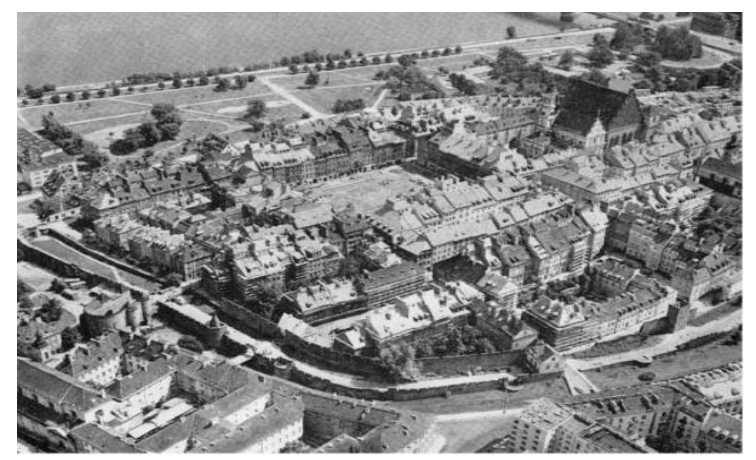

شكل 5 الساحة الرئيسة لمدينة وارسو بعد إعادة الإعمار.

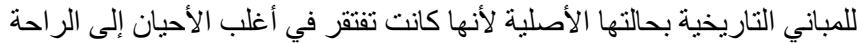

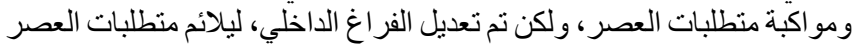

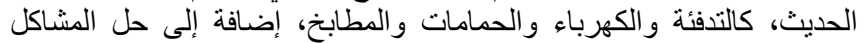

الداخلية السابقة كالتهوية والنّة الإضاءة ولاهو الرطوبة.

الكثف عن أقدم طبقات التاريخ: نم الكثف عن طبقات أقدم في البناء و النسيج

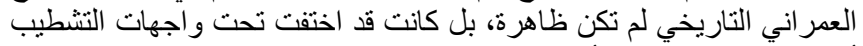

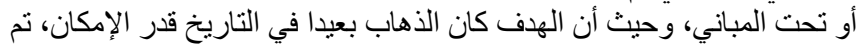

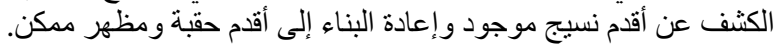

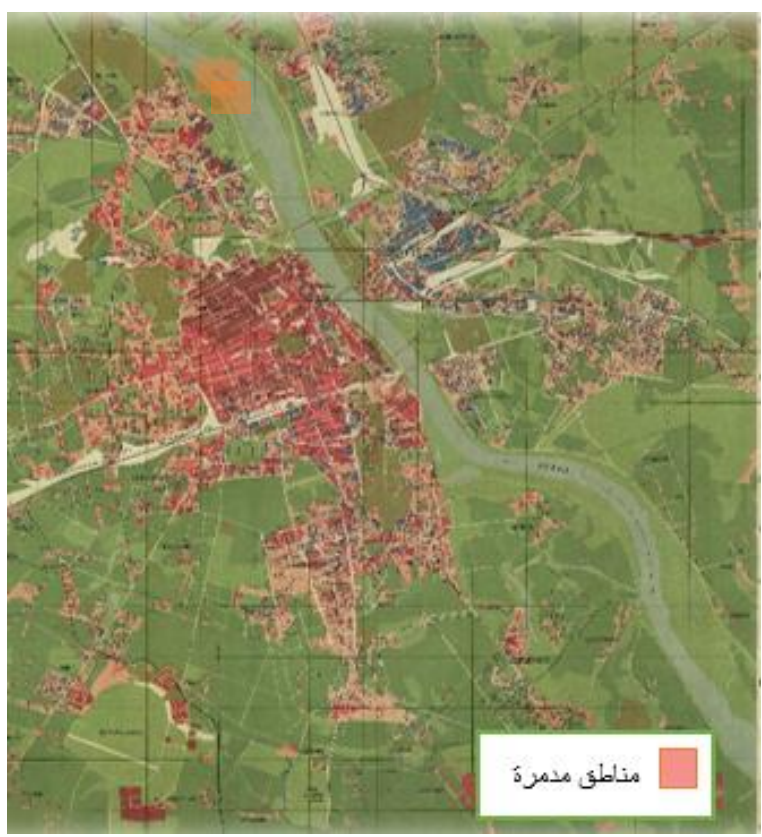

شكل 1 خريطة توضح الدمار الذي أصاب مدينة وارسو أثناء الحرب العالمية الثانية المصدر: : [27]

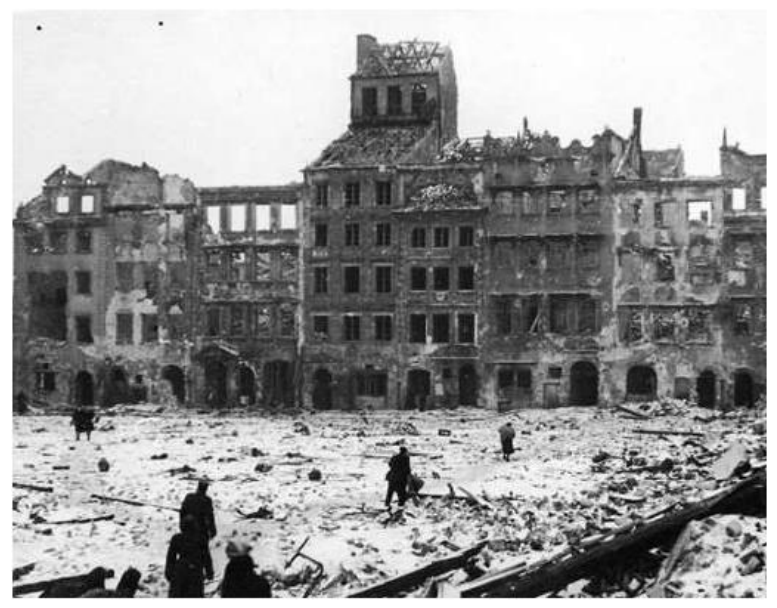

شكل 2 المباني في مدينة وارسو بعد الحرب.

[30]:المصن

تعزيز مؤسسات المجتمع للمساهمة في إعادة الإعمار : انهارت الحكومة الرمات الرسمية

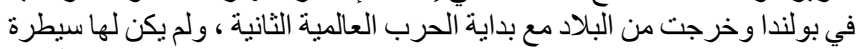

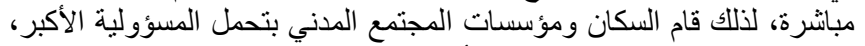

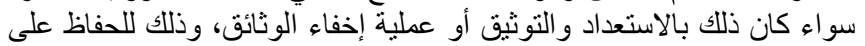

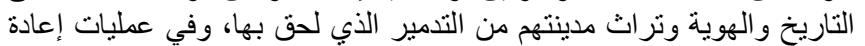

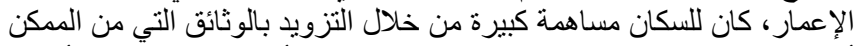

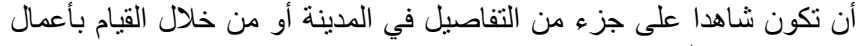

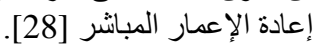




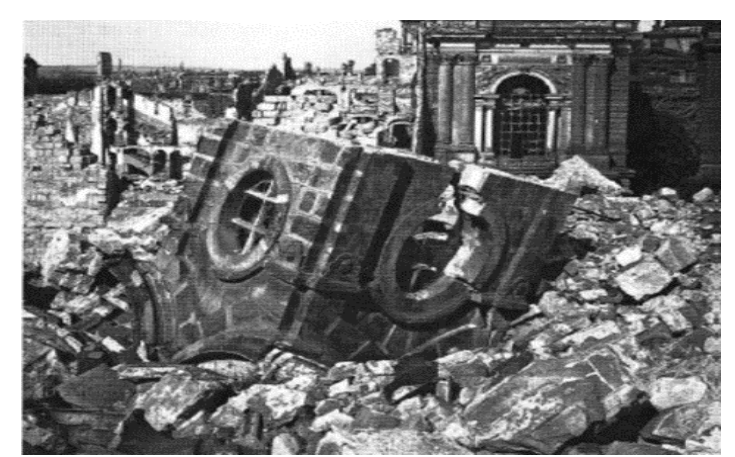

شكل 6 الأحجار التي نم إنقاذها وإعادة استخدامها في إعادة الإعمار [28]

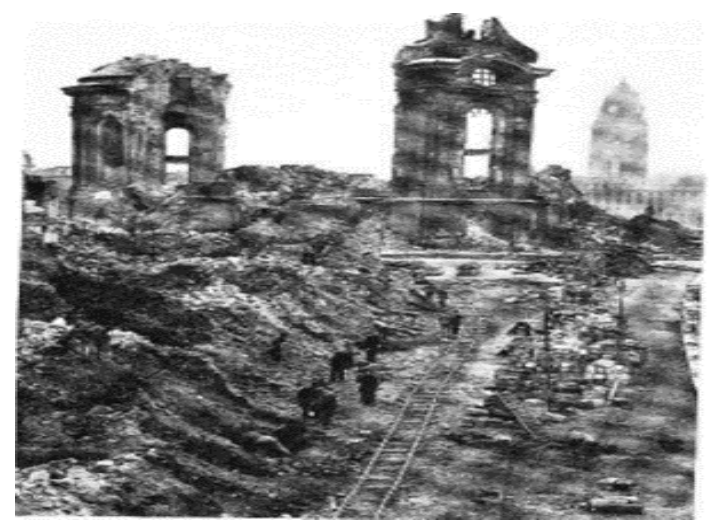

شكل 7 تشكيل سلاسل بشرية ومسار ات لتمرير الحجارة القابلة للاستخدام إلى نقطة

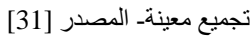
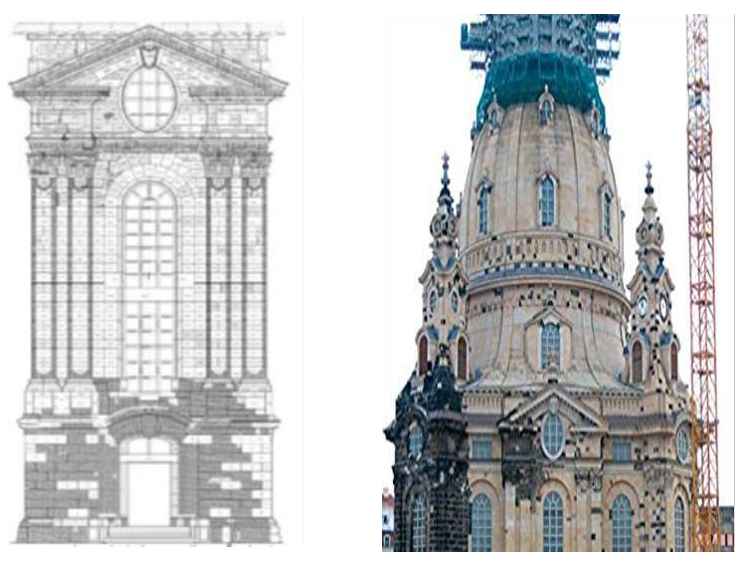

شكل 8 يوضح استخدام و إسقاط الأحجار القديمة مع الجديدة.

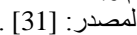

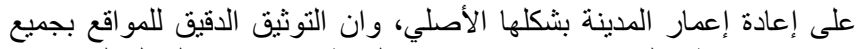

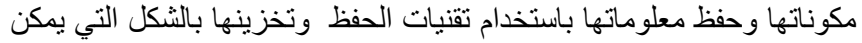

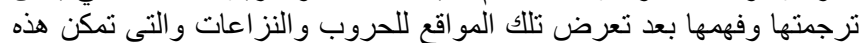

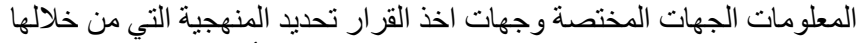

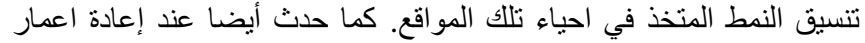

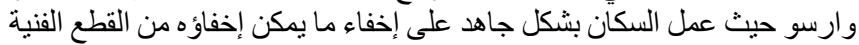

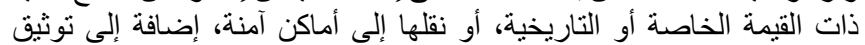

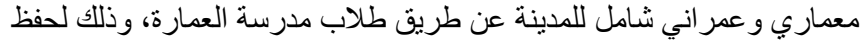

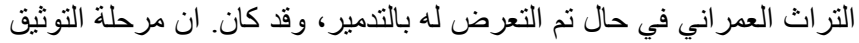

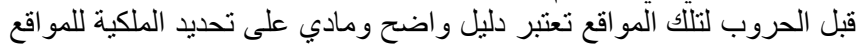

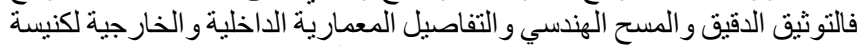
السيدة العذر اء ساعدت في تحديد موقع الكنيسة الأصلي بالر غم من الدمار الثـامل
2-2 تجربة إعادة إعمار كنيسة السيدة العذراء درسدن/ألمانيا

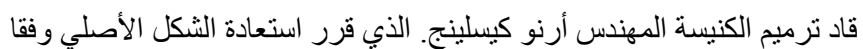

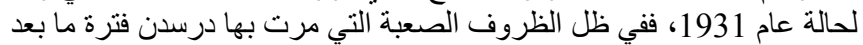

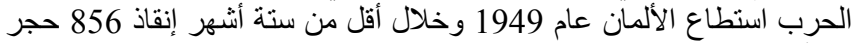

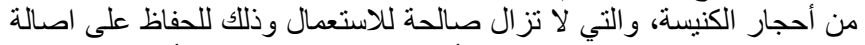

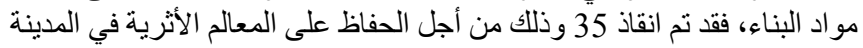

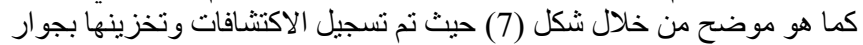

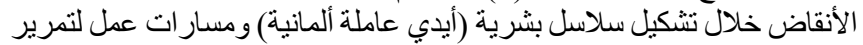

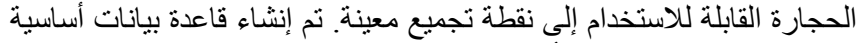

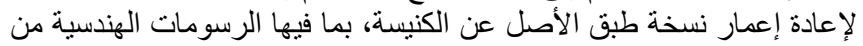

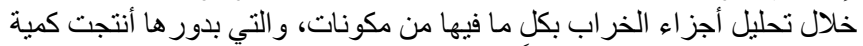

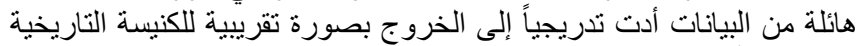

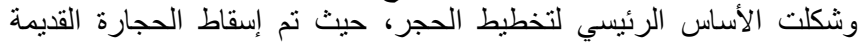

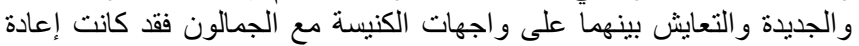

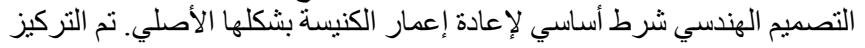

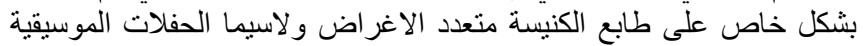

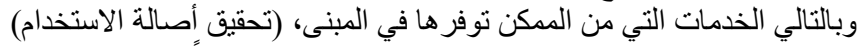

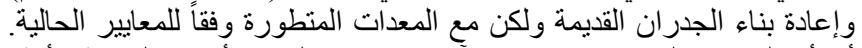

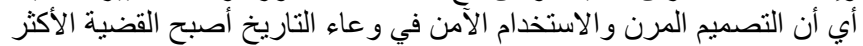

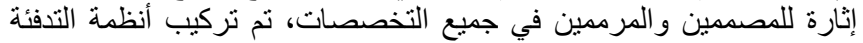

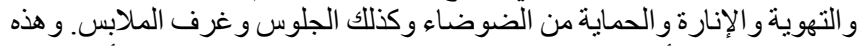

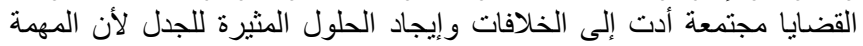

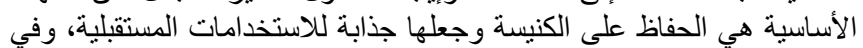

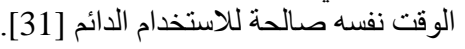
وتم تحقيق الأصالة في إعادة البناء بالآتي:

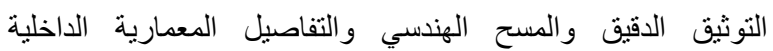

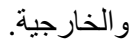

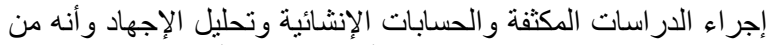

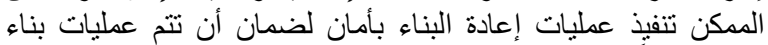

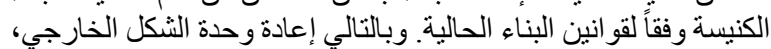

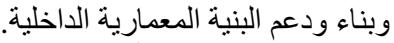

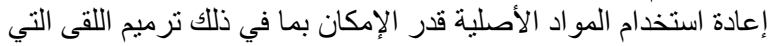

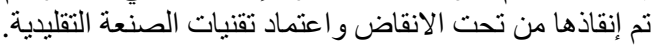

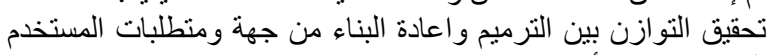

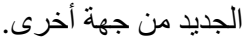

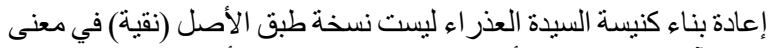

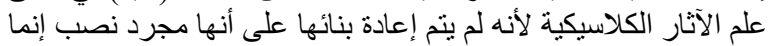

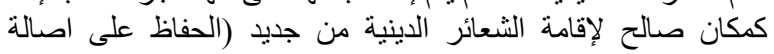

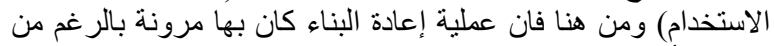
تحقيق الأصالة في الثكل و التصميم و المو اد و الاستخدام. السلبيات و العوائق التي واجهت عمليات إعادة الإعمار : عدم إز الة الأنقاض بشكل سريع وكامل. لا يوجد سياسة إعمار تعكس الانتماء النداء الوطني. تدخلات تمويلية و إدارية لفرض سيارسة الأنياء العمار معينة.

الإيجابيات في عملية الإعمار:

مشاركة السكان المحليين في الإعمار.

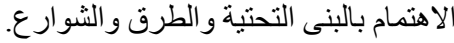

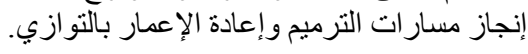

من خلال الإطار النظري ودراسة التجربتين السابقتين كإطار تحليلي تم

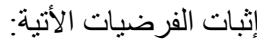

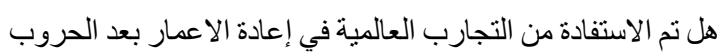

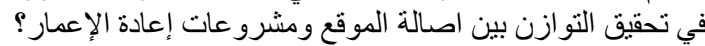

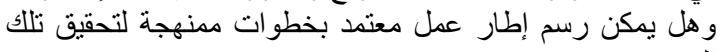

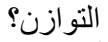
لاحظنا أن مرحلة الاستعداد قبل وقوع الكارئ الكارثة تعتبر من أهم المر احل التي

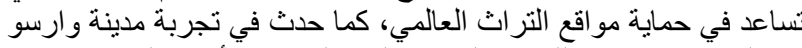

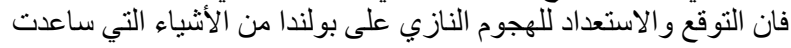




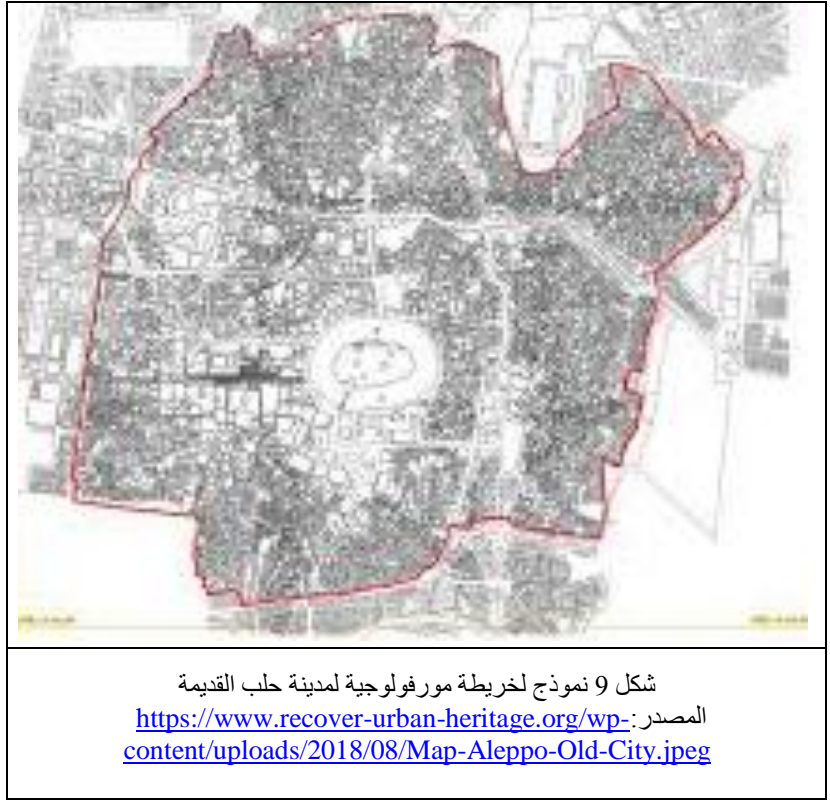

الأرشيف الرقمي

ويتم استخدامه كذاكرة مهمة للمعلومات لظروف ما قبل النزاع. ومن خلاله

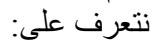

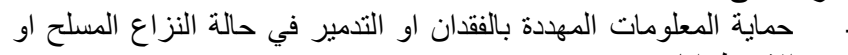
الاضطر ابات.

تسجيل مدى انتهاكات الاتفاقيات الدولية المتعلقة بحماية التراث الثقافي من

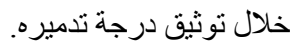

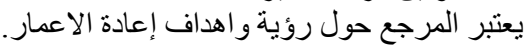

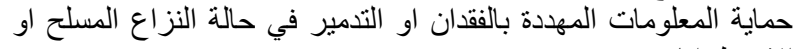
الاضطر ابات.

يعتبر مصدر للمعلومات المتاحة لترميح المباني و المجتمعات المتضررة المبات

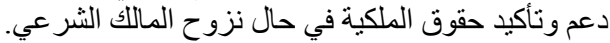

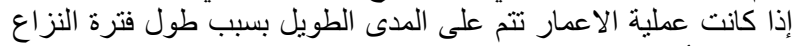

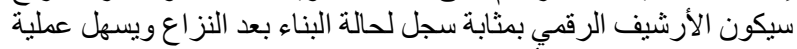

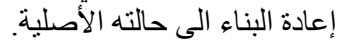

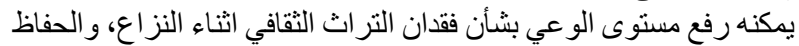

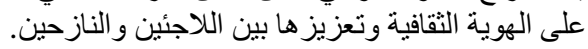

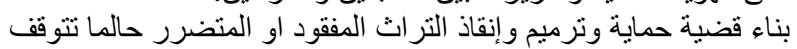
الاعمال العدائية.

$$
\text { النماذج ثلاثنية الأبعاد }
$$

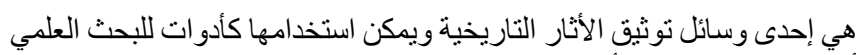

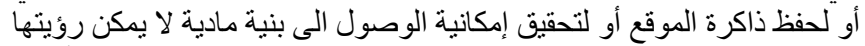

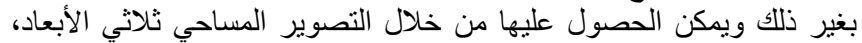

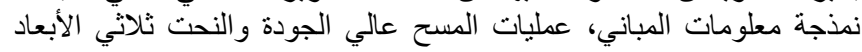

$$
\text { المعلومات الأثرية }
$$

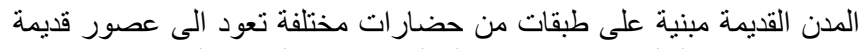

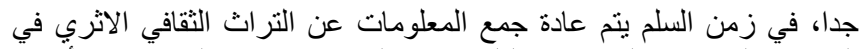

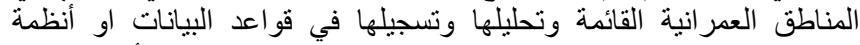

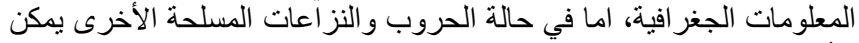

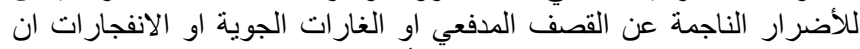

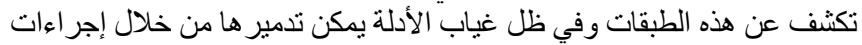

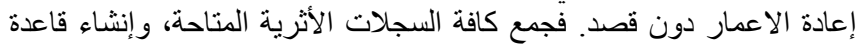

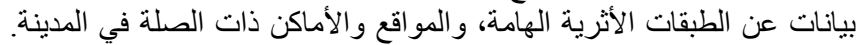

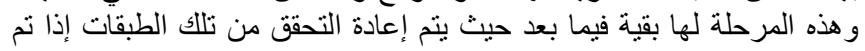

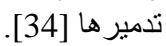

للمدينة. إعادة استخدام المواد الأصلية قدر الإمكان في إعادة الاعمار في كلا

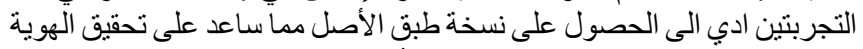

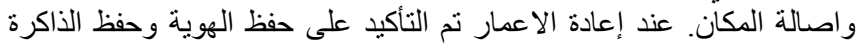

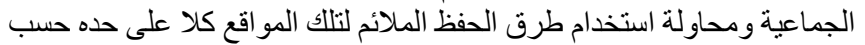

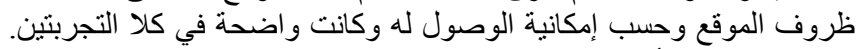

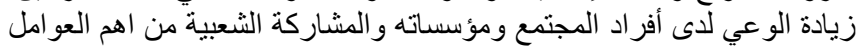

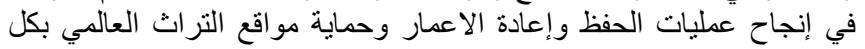

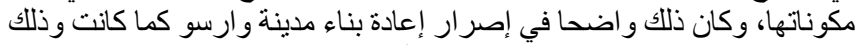

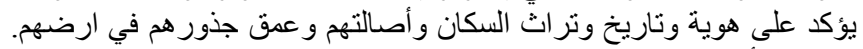

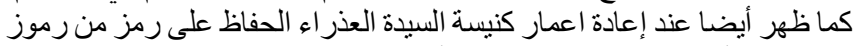
المدينة مما أدى الى التفكير في إعادة تأهيل و إعمار الوسط التئل التاريخي المحيط.

3.

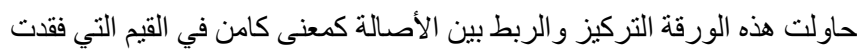

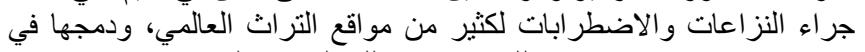

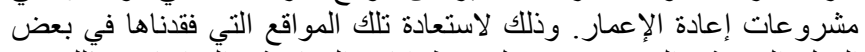

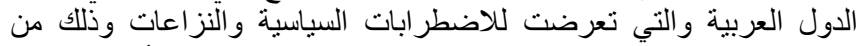

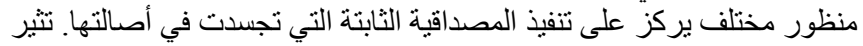

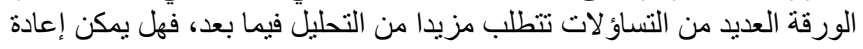

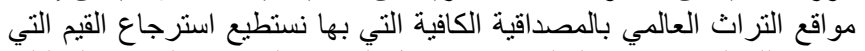

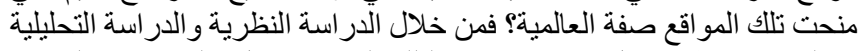

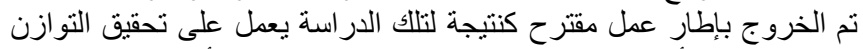

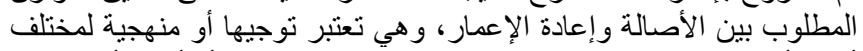

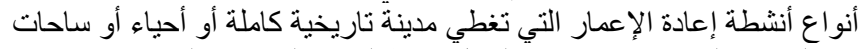

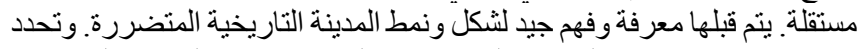

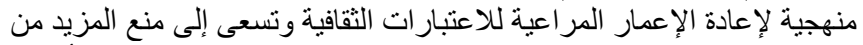

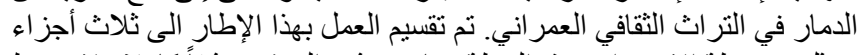

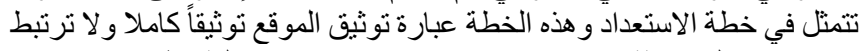

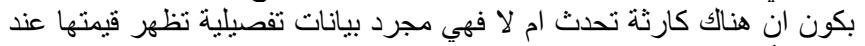

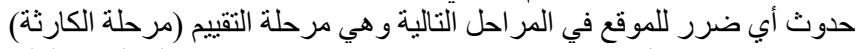

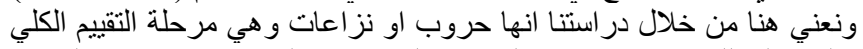

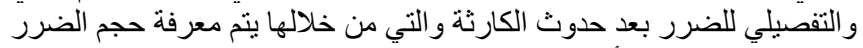
الذي لحق بالموقع ثم تأتي مرحلة إعادة الإعمار.

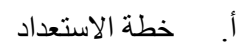

هذه اول مرحلة تتضمن بعض الارشادات وتكون كمرجع متكامل وتفصيلي الإني

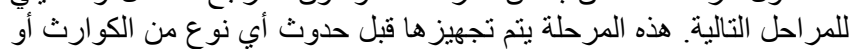

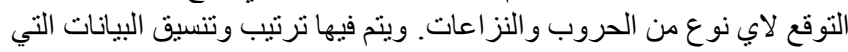

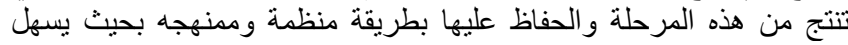

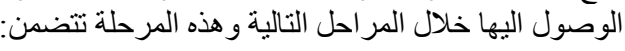

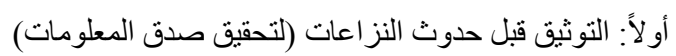

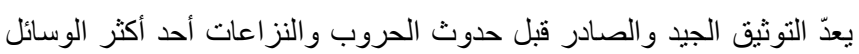

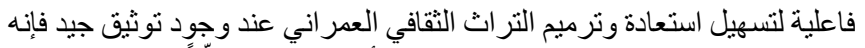

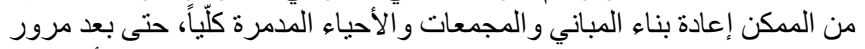

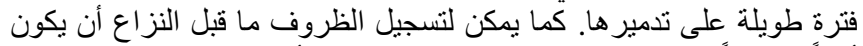

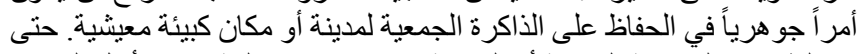

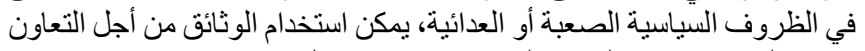

وحشد الدعم لاستعادة التراث المفقود. ويتم من خلال النسل [32].

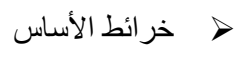

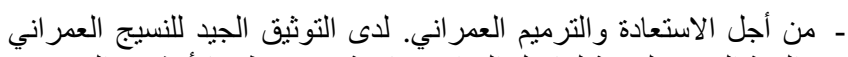

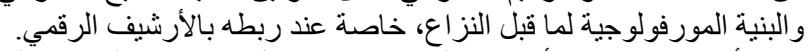

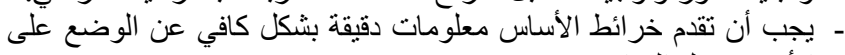
الأرض، قبل النز أنماع.

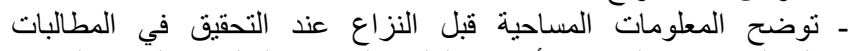

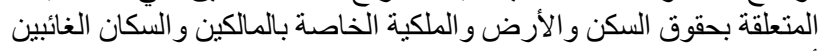

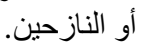

- توفر خر ائط الأساس نقطة انطلاق للتخطيط من أجل إعادة بناء مركز المدينة التاريخية بأكمله. 
والإصلاح، وتتيح تحديد المتطلبات الفنية والمالية للقطاعات والأصول الفردية

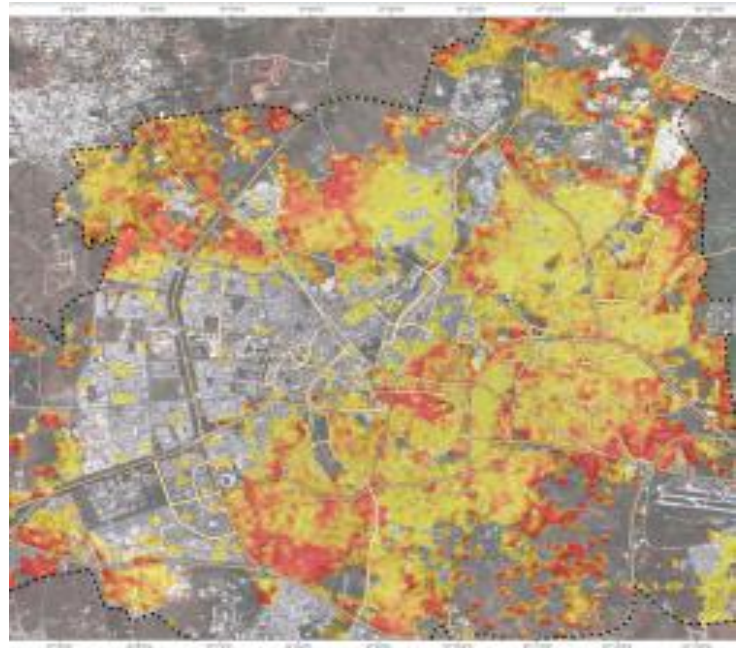

شكل 12 صوره ملتقطة بالأقمار الصناعية تبين مدى الضرر - حلب- سوريا https://www.recover-urban-heritage.org/basic-maps. المصدئ

$$
\text { تقييم أضرار المعالم التاريخية }
$$

و هو بمثابة إلقاء نظرة فاحصة على حالة المباني الفردية، أو مجمعات الأبنية،

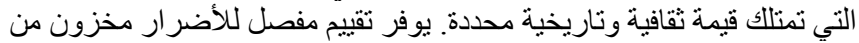

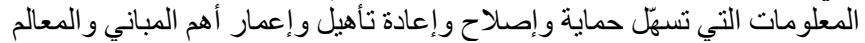

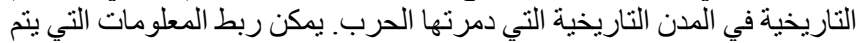

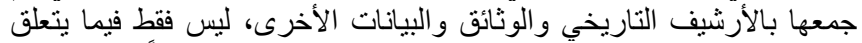

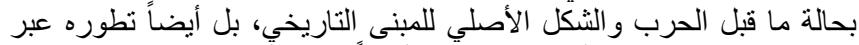

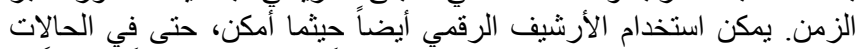

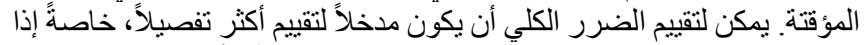

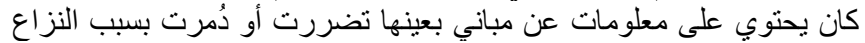

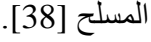

$$
\text { إنقاذ مو اد البناء التاريخية. (لتحقيق اصالة المواد) }
$$

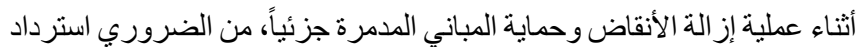

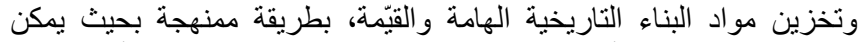

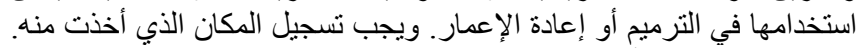

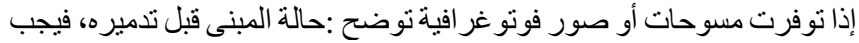

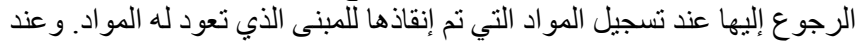

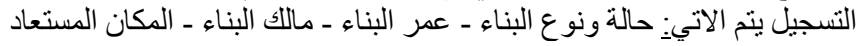

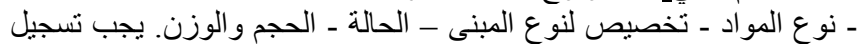

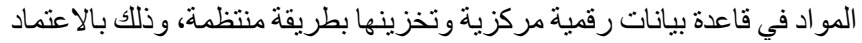

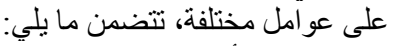

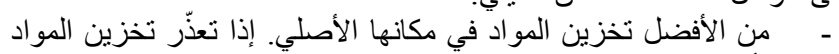

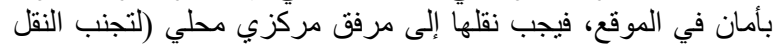

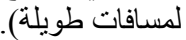

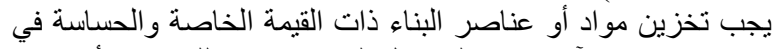

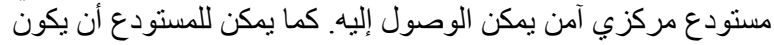

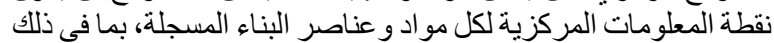

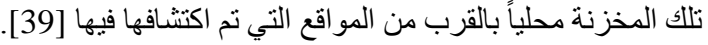

$$
\text { ج. مرحلة التخطيط و إعادة الإعمار }
$$

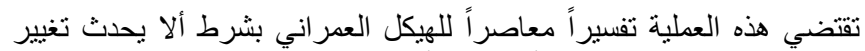

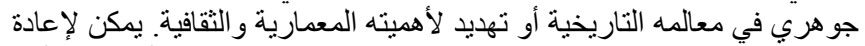

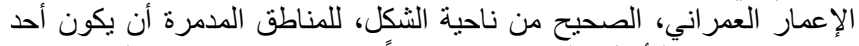

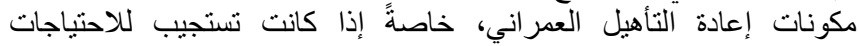

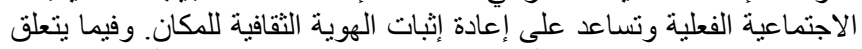
بالمباني المفردة، فإن إعادة التأهيل تستلزم إعادة استخدات التهام هياكل الأبنية التاريخية

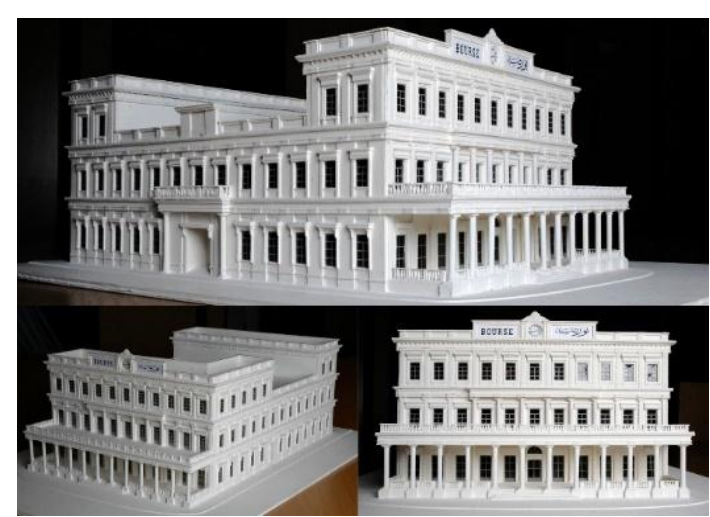

شكل 10 نموذج ثلاثي الابعاد لمبنى البورصة بالإسكندرية الذي تم تدميره

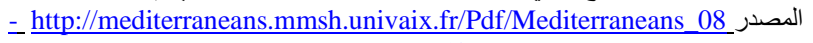
09 07.pdf

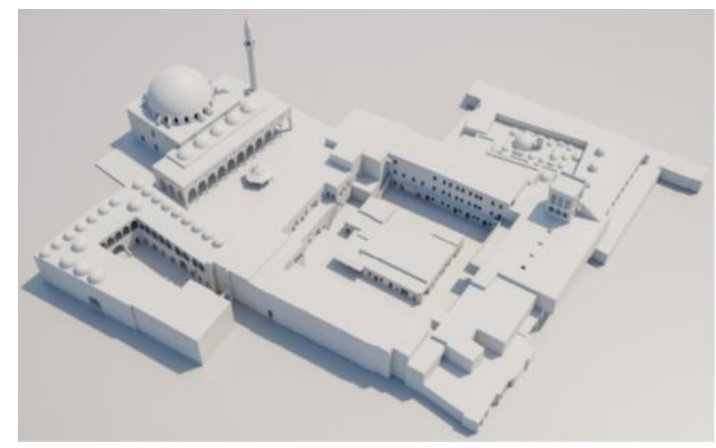

شكل 11 نموذج ثلاثي الأبعاد لسوق حلب الذي تم تدميره

https://www.recoverurbaheritage.org/3d-modeling/?lang=ar المصدي ندون

$$
\text { ب. مرحلة تقييم الأضرار }
$$

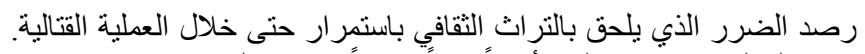

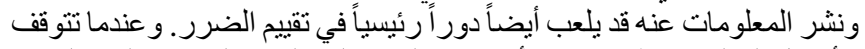

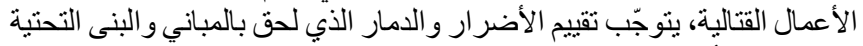

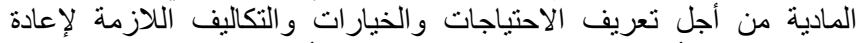

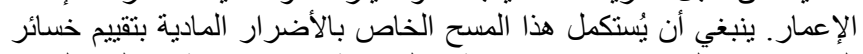

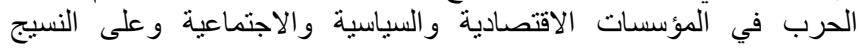

الاجتماعي [35].

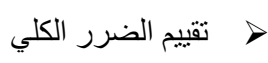

هو تقييم الأضر ار التي لحقت بالمباني التاريخية والبنية العمر انية الحساسة للمدن

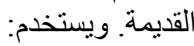

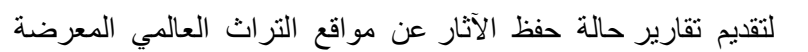
للخطر إلى مركز اليونسكو للتراثر الثار العالمي.

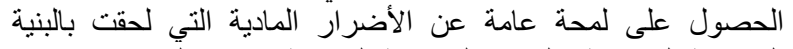

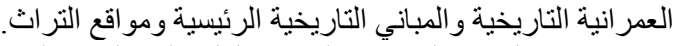

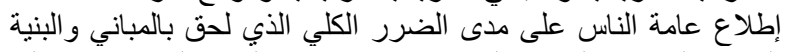

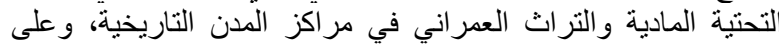
الحاجة إلى إعادة الإعمار.

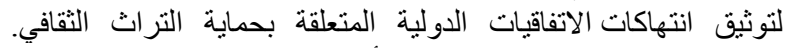

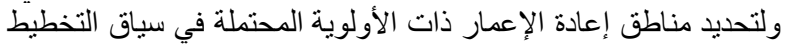

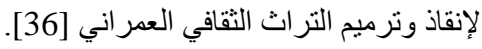

$$
\text { > }
$$

تركز على تقييم الأضرار التي تعرضت لها البنية التحتية مثل شبكات المياه

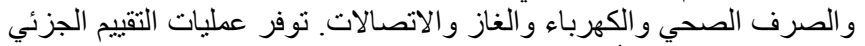

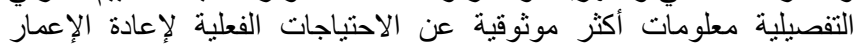


تمكننا من تحقيق مصداقية القيم الملموسة والغير ملموسة، و التي كانت تحملها

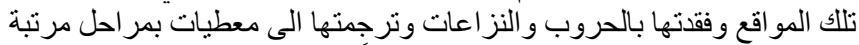

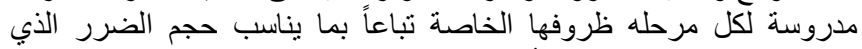

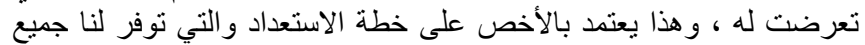

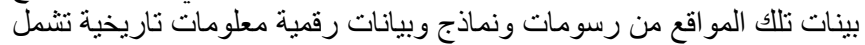

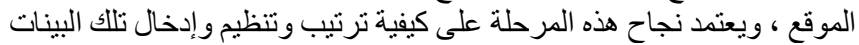

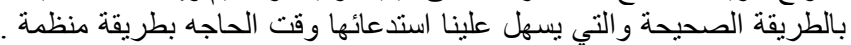

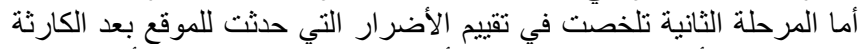

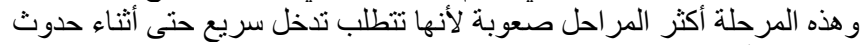

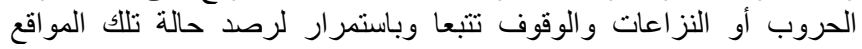

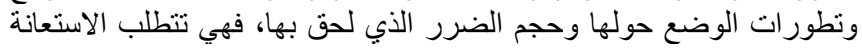

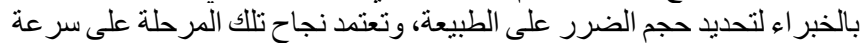

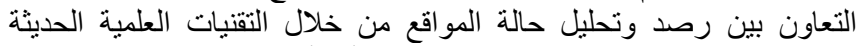

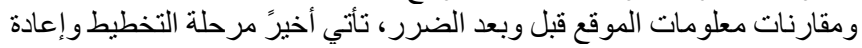

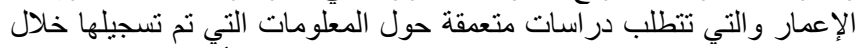

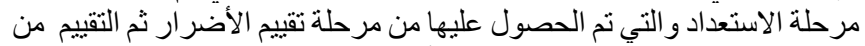

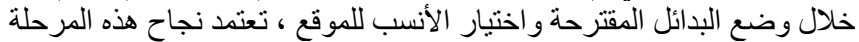
بكفاءة المعنيين من الخبر اء في كل مجالات تللك المرحلة.

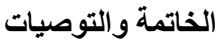

التراث العالمي مورد هش غير قابل للتجديد أو الاستبدال والهدف من حماية

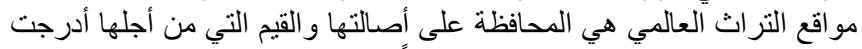

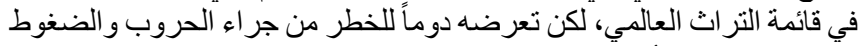

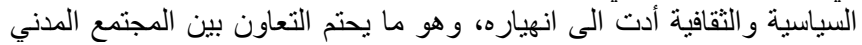

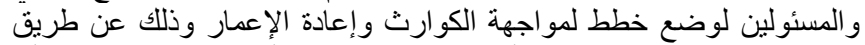

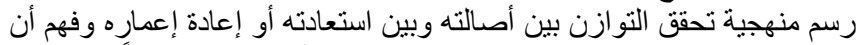

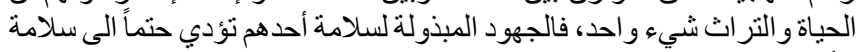
الأخر . بذلك نكون على استعداد لمو اجهة ما قد يحدث واستعادة ما فقد من تلكئك

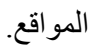

توصيات على المستوى الدولي:-

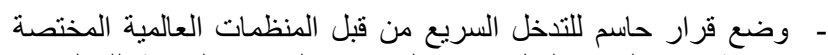

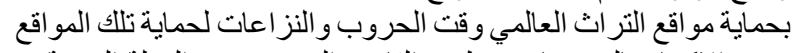

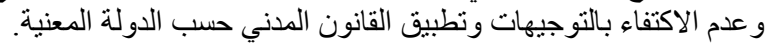

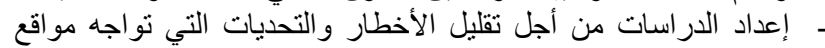

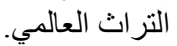

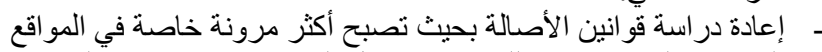

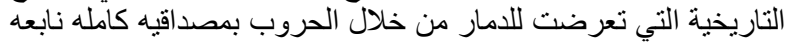
من الإحساس بالمكان.

\section{توصيات على المستوى القومي:-}

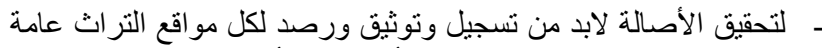

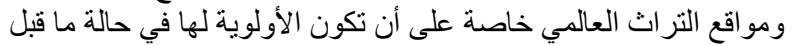

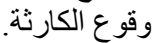
- لابد أن يكون التوثيق بشكل تفصيلي ويمتد ليشمل كل عناصر البيئة

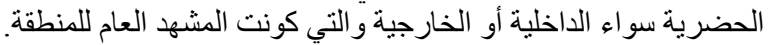

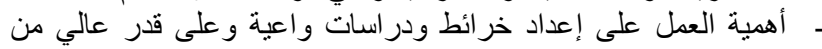

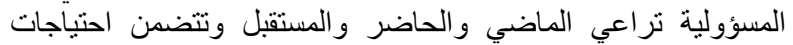

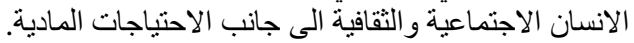

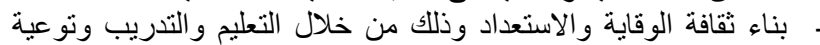
الناس و الفاعلين.

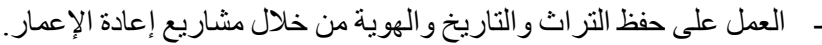

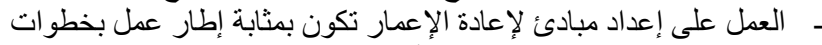

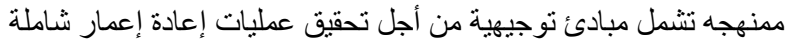
تهتم بجميع الجو انب بشكل عام وتحقيق الاصنالة و الحفاظ على الهوية بشكل إعلى

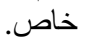
- الاستفادة من التقدم التكنولوجي وبرامج الحاسوب في منهجية الحفظ و التوثيق الجيد.
على نحو تكيّفي. فهذه العملية تجمع بين الاستعادة المادية والوظائف المفيدة

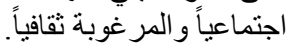
الالنز امات و الخطط السابقة للنز اعات

تعد الالتزامات السابقة للنزاع الناشئة من الاتفاقيات الدولية المتعلقة بالتراث

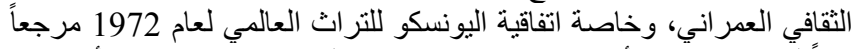

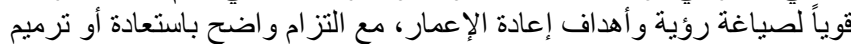

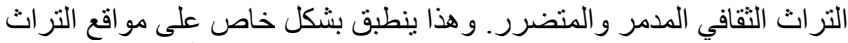

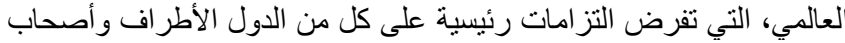

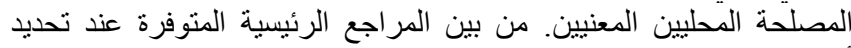
هداف إعادة الإعمار ما يلي: الإلين

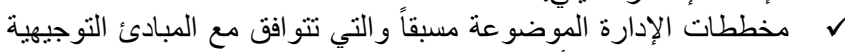
التنفيذية لليونسكو بشأن تنفيذ اتفاقية التراثر الثئ العالمي.

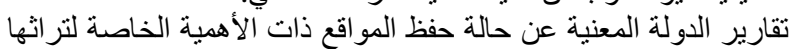

العمر اني والمدرجة في قائمة التراث العالمي المعرض المات للخطر [40].

$$
\text { تحقيق أصالة التصميم في إعادة الإعمار }
$$

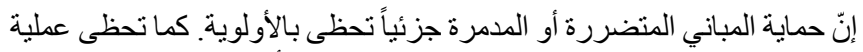

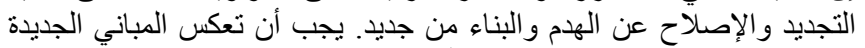

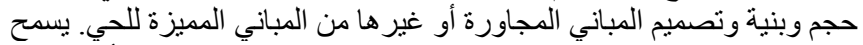

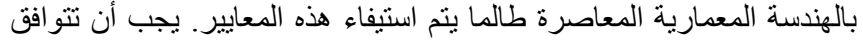

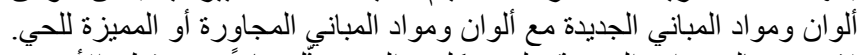

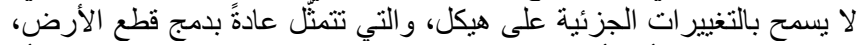

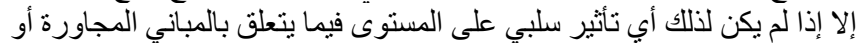
المباني الأخرى المميزة للحي.

$$
\text { إرشادات لاسترجاع مركز المدينة التاريخية }
$$

المدينة التاريخية القديمة هي أهم الأصول الثقافية و التجارية و السياحية، فهي تمنح

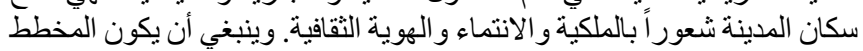

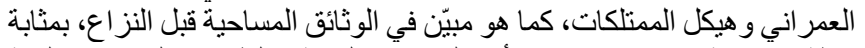

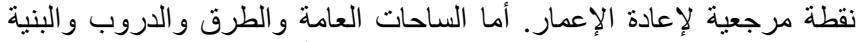

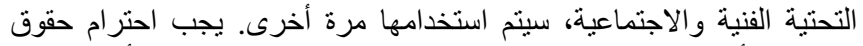

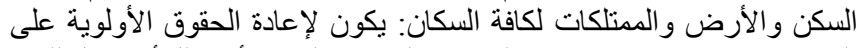

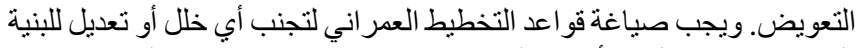

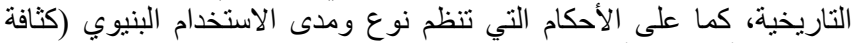

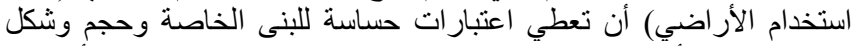
المباني وينبغي أن تطبّق القوانين المبني المتمدة على المباني التي لم تمتنل لأحكامها

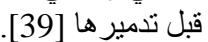

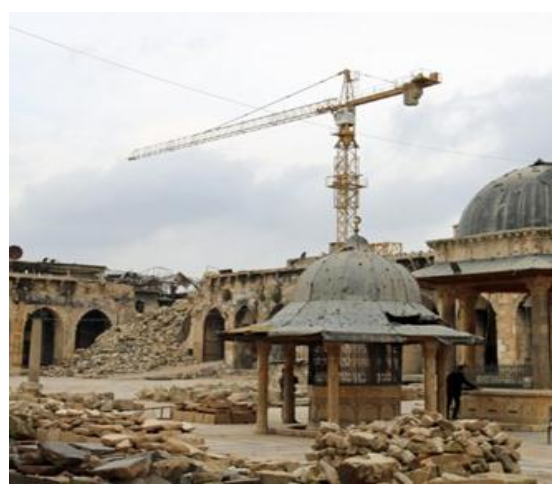

شكل 13 الجامع الاموي بعد التدمبر - سوريا.

https://www.recoverurbanheritage.org/reconstructio:المعليا. nguidelines/?lang=ar

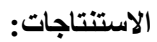

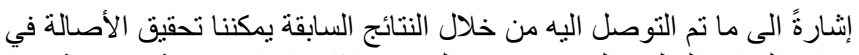

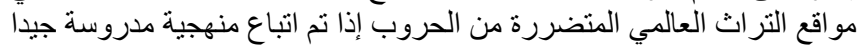


in conservation of historic buildings," in Cib 2010 World Conference, Building a Better World, 2010, pp. 188-198.

[14] G. Boccardi, "Authenticity in the heritage context: a reflection beyond the nara document," Hist. Environ. Policy Pract., vol. 10, no. 1, 2019, pp. $4-18$,.

[15] U. ICOMOS, "Charter for the Conservation of Historic Towns and Urban Areas (Washington Charter, 1987)," 1987.

[16] B. McKercher and H. Du Cros, Cultural tourism: The partnership between tourism and cultural heritage management. Routledge, 2002.

[17] A. Abdel Tawab, Evaluating the appropriateness of new development introduced inside ensembles of modern heritage to their heritage qualities (conference presentation). 2018.

[18] T. Fejérdy, "Authenticité dans la restauration des monuments historiques," in Nara conference on authenticity in relation to the World Heritage Convention. Proceedings, 1995, pp. 211- 216.

[19] H. C. Alberts and H. D. Hazen, "Maintaining authenticity and integrity at cultural world heritage sites," Geogr. Rev., vol. 100, no. 1, 2010, pp. 56-73.

[20] G. Fangi, "Aleppo-Before and after," Int. Arch. Photogramm. Remote Sens. Spat. Inf. Sci, 2019, pp. 333-338.

[21] M. K. Lindell, "Recovery and reconstruction after disaster," Encycl. Nat. hazards, vol. 8, 2013, pp. 12-824.

[22] M. Petzet, "Principles of preservation: an introduction to the international charters for conservation and restoration 40 years after the venice charter," 2004.

[23] A. kozien, "protection of the authenticity and integrity of monuments as a determinant affecting the activities of entrepreneurs-the owners of monuments," econ. soc. dev. b. proc., 2021, pp. 21-31.

[24] N. Stanley-Price, "The reconstruction of ruins: principles and practice," Conserv. Princ. dilemmas uncomfortable truths, 2009, pp. 32-46.

[25] R. W. Khalaf, "World Heritage policy on reconstruction: from exceptional case to conservation treatment," Int. J. Cult. Policy, vol. 25, no. 7, 2019, pp. 871-885.

[26] M. Al Aloul, "The destruction of cultural heritage by warfare and reconstruction strategies: lessons learned from case studies of rebuilt cities." University of Florida, 2007.

[27] https://i.redd.it/jggrh5h58w201.jpg

[28] S. Jankowski, "Warsaw: destruction, secret town planning, 1939-44, and postwar reconstruction," in Rebuilding Europe's bombed cities, Springer, 1990, pp. 77-93.

[29] https://whc.unesco.org/en/list/30/

[30] T. Jelenski, "Practices of Built Heritage Post-Disaster Reconstruction for Resilient Cities," Buildings, vol. 8, Mar. 2018, pp. 53, doi: 10.3390/buildings 8040053 .

[31] B. Collins et al., "The Dresden Frauenkirche-rebuilding the past," BAR Int. Ser., vol. 598, 1995, pp. 19.

[32] https://www.recover-urban-heritage.org/documentation/?lang=ar

[33] https://www.recover-urban-heritage.org/3d-modeling/?lang=ar,

[34] https://www.recover-urban-heritage.org/archaeologicalinformation/?lang=ar,

[35] https://www.recover-urban-heritage.org/damage-assessment/?lang=ar,

[36] https://www.recover-urban-heritage.org/overall-damage/?lang=ar,

[37] https://www.recover-urban-heritage.org/sectoral-damage/?lang=ar,

[38] https://www.recover-urban-heritage.org/historic-monument/?lang=ar,

[39] https://www.recover-urban-heritage.org/reconstructionguidelines/?lang=ar

[40] https://www.recover-urban-heritage.org/international-conventions-onurban-cultural-heritage/?lang=ar,
- الحفاظ بقدر الإمكان على المواد الأساسية والقطع الفنية القيمة لإعادة

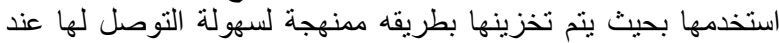

إعادة الإعمار.

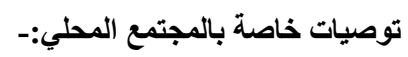

- أهمية المشاركة الثعبية وخصوصا المختصين في اثارة النقاش و المساهمة في اعداد الاستر اتيجيات.

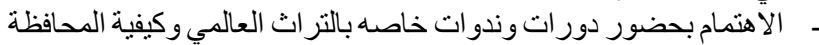

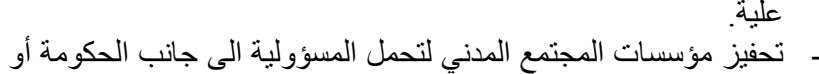
تحمل المسؤولية كاملة في أوقات الكوات الكوارث

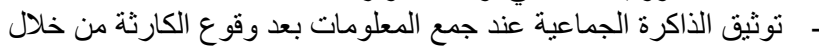

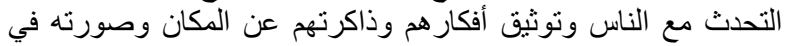

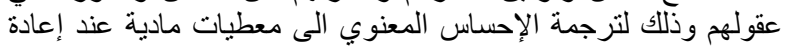

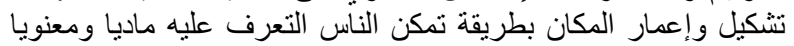

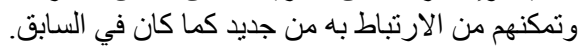

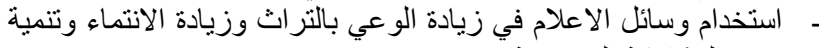
روح المشاركة المجتمعية.

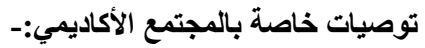

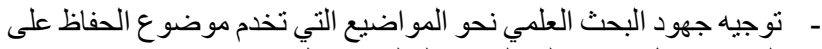

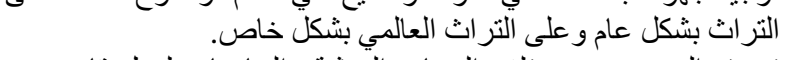

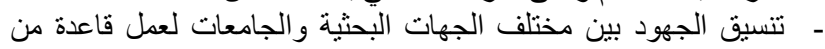
البيانات و الخر ائط التي تخدم مشاريع الحفاظ.

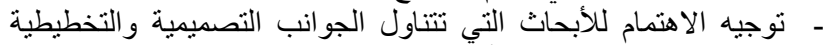

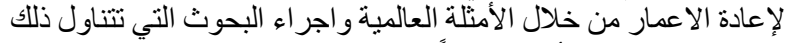

للاستفادة منها بشكل أكثر اتساعلاً.

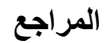

[1] J. Lawless and K. Silva, "Towards an Integrative Understanding of 'Authenticity' of Cultural Heritage: An Analysis of World Heritage Site Designations in the Asian Context," J. Herit. Manag., vol. 1, Dec. 2016, pp. 148-159, doi: 10.1177/2455929616684450.

[2] B. M. Feilden and J. Jokilehto, "Management guidelines for world cultural heritage sites," 1998.

[3] S. Labadi, "World Heritage, authenticity and post-authenticity," Herit. Glob., 2010, pp. 66-84.

[4] L. Meskell, "States of conservation: protection, politics, and pacting within UNESCO's World Heritage Committee," Anthropol. Q., 2014, pp. 217-243.

[5] J. Pendlebury, M. Short, and A. While, "Urban World Heritage Sites and the problem of authenticity," Cities, vol. 26, no. 6, 2009, pp. 349358.

[6] M. G. Abdelmonem, "Architectural and urban heritage in the digital age: dilemmas of authenticity, originality and reproduction," Int. J. Archit. Res. ArchNet-IJAR, vol. 11, no. 3, 2017, pp. 5-15.

[7] A. Potter, The authenticity hoax: How we get lost finding ourselves. Emblem Editions, 2011.

[8] E. Waterton and S. Watson, The Palgrave handbook of contemporary heritage research. Springer, 2015.

[9] R. W. Khalaf, "A viewpoint on the reconstruction of destroyed UNESCO Cultural World Heritage Sites," Int. J. Herit. Stud., vol. 23, no. 3, 2017, pp. 261-274.

[10] S. Jones, "Experiencing authenticity at heritage sites: Some implications for heritage management and conservation," Conserv. Manag. Archaeol. Sites, vol. 11, no. 2, 2009, pp. 133-147.

[11] J. Garcia-Esparza, "Clarifying dynamic authenticity in cultural heritage. A look at vernacular built environments.," ICOMOS University Forum, 2018, vol. 1, pp. 1-12.

[12] J. Jokilehto, "The context of the Venice Charter (1964)," Conserv. Manag. Archaeol. sites, vol. 2, no. 4, 1998, pp. 229-233.

[13] C. Alho, A. Morais, J. Mendes, and A. Galvao, "Authenticity criteria 


\section{Abstract}

-Nations are based over time on their original history, so anyone who has no past has no present. Cultural heritage is one of the clearest physical images of heritage that makes history for the human being and creates an urban identity for him. On the contrary, the erasure of material history is one of the entrances to the erasure of nations, and this is what global cultural heritage is facing these days because of wars and armed conflicts. With the advancement of the war machine that destroyed entire historic sites or cities, reconstruction became a dream. Even when some countries resorted to restoring what had been destroyed, much of its authenticity was lost. Realizing the authenticity of the heritage after its destruction through reconstruction projects is a complex process that needs a strong strategy and an organized framework to benefit from previous successful experiences in reconstruction. Consequently, that study aimed, in both its theoretical and analytical parts, to come up with a framework that achieves a balance between the concept of authenticity adopted through international agreements and rebuilding those sites so that they do not lose their exceptional global value. 